\title{
Generation and Propagation of Tsunami by a Moving Realistic Curvilinear Slide Shape with Variable Velocities in Linearized Shallow-Water Wave Theory
}

\author{
Hossam Shawky Hassan ${ }^{1}$, Khaled Tawfik Ramadan ${ }^{1}$, Sarwat Nageeb Hanna ${ }^{2}$ \\ ${ }^{1}$ Department of Basic and Applied Science, College of Engineering \& Technology, Arab Academy for Science, \\ Technology and Maritime Transport, Alexandria, Egypt \\ ${ }^{2}$ Department of Engineering Mathematics and Physics, Faculty of Engineering, Alexandria University, \\ Alexandria, Egypt \\ E-mail:hossams@aast.edu \\ Received February 8, 2010; revised April 5, 2010; accepted April 13, 2010
}

\begin{abstract}
The process of tsunami evolution during its generation under the effect of the variable velocities of realistic submarine landslides based on a two-dimensional curvilinear slide model is investigated. Tsunami generation from submarine gravity mass flows is described in three stages. The first stage represented by a rapid curvilinear down and uplift faulting with rise time. The second stage represented by a unilaterally propagation in the positive $\mathrm{x}$-direction to a significant length to produce curvilinear two-dimensional models represented by a depression slump, and a displaced accumulation slide model. The last stage represented by the time variation in the velocity of the accumulation slide (block slide). By using transforms method, Laplace in time and Fourier in space, tsunami waveforms within the frame of the linearized shallow water theory for constant water depth are analyzed analytically for the movable source model. Effect of the water depths on the amplification factor of the tsunami generation by the submarine slump and slide for different propagation lengths and widths has been studied and the results are plotted. Comparison of tsunami peak amplitudes is discussed for different propagation lengths, widths and water depths. In addition, we demonstrated the tsunami propagation waveforms after the slide stops moving at different propagation times.
\end{abstract}

Keywords: Near-Far Field Tsunami Amplitudes, Shallow Water Theory, Water Wave, Submarine Slumps and Slides, Laplace and Fourier Transforms

\section{Introduction}

Tsunamis are surface water waves caused by the impulsive perturbation of the sea. Apart from co-seismic sea bottom displacement due to earthquakes, subaerial and submarine landslides can also produce localized tsunamis with large and complex wave runup especially along the coasts of narrow bays and fjords. In recent years, significant advances have been made in developing mathematical models to describe the entire process of generation, propagation and run-up of a tsunami event generated by seismic seafloor deformation, [1-3].

Tsunamis are also generated by other mechanisms such as submarine landslides, which often accompany large earthquakes, and can disturb the overlying water column as sediment and rock slump down slope. The common mechanisms for triggering failure of submarine slopes are over-steepening due to rapid deposition of sediments, generation of gas created by decomposition of organic matter, storm waves, and earthquakes, which are the major cause of landslides on continental slopes, [4]. The failed material is driven by the gravity forces. Any type of geophysical mass flow which includes a wide range of ground movement such as debris flows, debris avalanches and rock falls can create submarine landslide which generate tsunamis. These phenomena rapidly displace large volumes of water, as energy from falling debris or expansion is transferred to the water into which the debris falls at a rate faster than the ocean water can absorb it. They have been named by the media as "mega-tsunami".

In terms of tsunami-generation mechanisms, two sig- 
nificant differences exist between submarine-landslide and coseismic seafloor deformation. First, the duration of a landslide is much longer and is in the order of magnitude of several minutes. Hence the time-history of the sea floor movement will affect the characteristics of the generated wave and needs to be included in the model. Secondly, the effective size of the landslide region is usually much smaller than the co-seismic seafloor-deformation zone. Consequently, the typical wavelength of the tsunamis generated by a submarine landslide is also shorter. Therefore, the frequency dispersion could be important in the wave-generation region.

The case of particular interest to this study is the mechanism of generation of tsunamis by submarine landsides. When a submarine landslide occurs the ocean-bottom morphology may be significantly altered, in turn displacing the overlying water. Waves are then generated as water gets pulled down to fill the area vacated by the landslide and to a lesser extent, by the force of the sliding mass. Submarine slides can generate large tsunami, and usually result in more localized effects than tsunami caused by earthquakes, [5].

Modeling of tsunami generation and propagation caused by submarine slides and slumps is a much more complicated problem than simulation of seismic-generated tsunami as the characteristics of a tsunami generated by a submarine landslides are mainly determined by the volume, acceleration, velocity and rise time of the slide motion. However, we constructed a realistic submarine landslide based on two-dimensional curvilinear slide model representing submarine slump and slide involved in the transform methods that may generate a tsunami neat the source region.

The speed at which the mass moves across the sea floor is critical for the wave heights attained. Very fast slides (debris-flows) generate tsunamis roughly as high as the slide is thick while very slow moving slides produce little or no tsunamis. However, where slides move at velocities close or equal to that of the tsunami being produced, they develop 'in phase', building the waves up to exceptional size.

Constant velocity implies that the slide starts and stops impulsively, i.e. the deceleration is infinite both initially and finally. Clearly, this is not true for real slides, and a more complex shape of the generated wave is expected.

Tsunami waves may be generated by a slide that starts from rest accelerates with the same maximum velocity and decelerate back to rest. Another morphological feature of underwater slides is that the mass often travels significant distances from the headwall scar before coming to rest. This indicates a rapid acceleration and large translational velocities. Hence, landslides sources are not impulsive and tsunami propagation starts while the forcing process is still in action. Rock slides plunging into fjords, lakes, or reservoirs are most often super-critical and can generate huge, destructive waves. Tsunamis may cause large oscillations in basins or fjords, causing a series of incident waves. In this study, we determine the possibility of similar trends for a submarine rock slide. We study slide motion and deformation at early times while the slide is still decelerating and while tsunami generation is still taking place. These two conditions are redundant because one can define the duration of tsunami generation by the characteristic duration of slide acceleration [5].

Previous studies of tsunami generation have been concerned with the surface elevations induced by the impulsive motion of an impermeable rigid bottom resulting from an undersea earthquake in one direction, [6-9] and in two directions, [10,11]. All these studies are taken into account solely for constant depth. It is obvious that the effects of ocean depth on tsunami amplitudes are significant. Therefore, in our work, the effects of ocean depth on tsunami amplitudes are analyzed. To determine the effects, 19 ocean depths ranging from 200-2000 m are studied.

In recent years, the results of numerical and analytical studies, simulating mechanism of tsunami caused by submarine landslides are discussed. Beisel, et al. [12] studied numerically the landslide mechanism of tsunami generation based on a complex of multi-parameters calculations with the help of algorithms. Lynett and Liu Philip [13] derived mathematically a full nonlinear model to describe the generation and propagation of water waves by a submarine landslide. The model consists of a depth-integrated continuity equation and momentum equations, in which the ground movement is the forcing function. This model is capable of describing wave propagation from relatively deep water to shallow water. They developed a numerical algorithm for the general fully nonlinear model. Jiang and Le Blond [14] investigated coupling of a submarine slide and the surface water waves it generates. They found that the two major parameters that determine the interaction between the slide and the water waves are the density of sliding material and the depth of initiation of the slide. Rzadkiewicz et al. [15] simulated an underwater landslide by introducing a two-phase description of sediment motion and using the volume of fluid (VOF) technique. Grilli and Watts [16] simulated waves due to moving submerged body using a boundary element method. Watts et al. [17] found that, assuming a realistic maximum displacement for a slump, everything else being equal, the slump generates smaller tsunami surface elevations and wave lengths than a corresponding slide, particularly in the far-field. With identical initial acceleration, tsunami characteristics of similar slides and slumps are initially similar, but differences arise since the acceleration phase lasts longer and the displacement is larger for a slide. Ataie-Ashtiani and Shobeyri [18] presented an incompressible-smoothed particle hydrodynamics (I-SPH) to simulate impulsive waves generated by landslides. Agustinus [19] investi- 
gated the breaking of long-waves propagating on shallow water with nonlinear friction on the sloping bottom. He found from his numerical results that in order to overcome wave breaking, nonlinear friction needs to be below certain level. Also, laboratory experimental studies on tsunami generation by a rigid solid body moving along the slope have been carried out by many researchers, [20-25].

The speed at which the mass moves across the sea floor is critical for the wave heights attained. Very fast slides (debris-flows) generate tsunamis roughly as high as the slide is thick while very slow moving slides produce little or no tsunamis. However, where slides move at velocities close or equal to that of the tsunami being produced, they develop 'in phase', building the waves up to exceptional size, [26]. The transient wave generation due to the coupling between the slide deformation and time variations in the moving velocity and the free surface has been considered by Trifunac et al. [27]. They discussed the effect of variable speeds of spreading of submarine slumps and slides on the near-field tsunami amplitudes. They illustrated the nature and the extent of variations in the tsunami waveforms caused by simple time variations of the frontal velocity of spreading for two-dimensional kinematic models of slides and slumps and compared the results with those for slide spreading with constant velocity. They found that the overall nature of the near-field tsunami amplitudes depended on the overall average speed of slumping and sliding remains unchanged, with respect to their previous studies in [6-9] with constant velocities of spreading. Hayir [28] investigated the motion of a submarine block slide with variable velocities and its effects on the near-field tsunami amplitudes. He found that the amplitudes generated by the slide are almost the same as those created by its average velocity. Both Trifunac et al. [27] and Hayir [28] used very simple kinematic source models represented by a Heaviside step functions for representing the generation and propagation of tsunami.

Therefore, in this paper, we concern about the tsunami amplitudes predicted in the near-field caused by time variation of a two-dimensional realistic curvilinear slide model. The curvilinear tsunami source model we considered based on available geological, seismological, and tsunami elevation. This model resembles the initial source predicted according to the initial disturbance recorded in $[29,30]$. At present, it is difficult to describe the details of movements at the ocean floor during sliding because of the paucity of high frequency inverse studies of ground deformations in the source area of past tsuna$\mathrm{mi},[31]$. Therefore, the basic idea is to illustrate the possible range of tsunami amplitudes using realistic source model.

The aim of this study is to determine how near-field tsunami amplitudes change according to variable velocities of submarine slide. We discuss the nature and the extent of variations in the peak tsunami waveforms caused by time variations of the frontal velocity and the deceleration for the two-dimensional curvilinear block slide model and compares the results with those for the slide moving with constant velocity. It will show how the changes in the slide velocity as function in time acts to reduce wave focusing. Numerical results are presented for the normalized peak amplitude as a function of the propagation length and width of the slump and the slide, the water depth, the time variation of moving velocity and the deceleration of the block slide.

The problem is solved using linearized shallow-water theory for constant water depth by transform methods (Laplace in time and Fourier in space), with the forward and inverse Laplace transforms computed analytically, and the inverse Fourier transform computed numerically by the Inverse Fast Fourier Transform (IFFT). Particular cases are compared with the results obtained by Trifunac et al. [9] and Hayir [28].

\section{Mathematical Formulation of the Problem}

Consider a three dimensional fluid domain $\Omega$ as shown in Figure 1. It represents the ocean above the submarine slump and slide area. It is bounded above by the free surface of the ocean $z=\eta(x, y, t)$ and below by the rigid ocean floor $z=-h(x, y)+\zeta(x, y, t)$, where $\eta(x, y, t)$ is the free surface elevation, $h(x, y)$ is the water depth and $\zeta(x, y, t)$ is the sea floor displacement function. The domain $\Omega$ is unbounded in the horizontal directions $\mathrm{x}$ and $\mathrm{y}$, and can be written as $\Omega=R^{2} \times[-h(x, y)$ $+\zeta(x, y, t), \eta(x, y, t)]$. For simplicity, before the earthquake, $h(x, y)$ is assumed to be a constant and the fluid is assumed to be at rest, thus the free surface and the

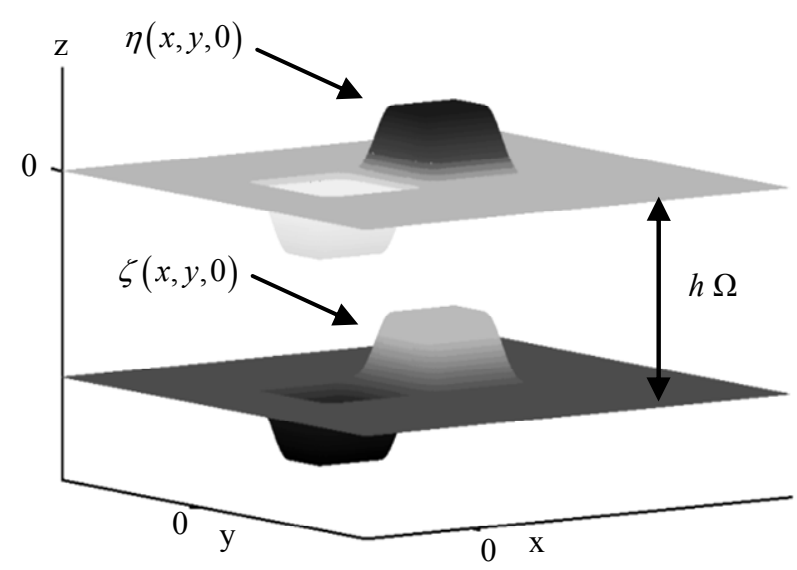

Figure 1. Fluid domain and coordinate system for a very rapid movement of the assumed source model. 
solid boundary are defined by $z=0$ and $z=-h$, respectively. Mathematically, these conditions can be written in the form of initial conditions: $\eta(x, y, 0)=$ $\zeta(x, y, 0)=0$. At time $t>0$ the bottom boundary moves in a prescribed manner given by $z=-h+$ $\zeta(x, y, t)$ The deformation of the sea bottom is assumed to have all the necessary properties needed to compute its Fourier transforms in $x, y$ and its Laplace transform in $t$. The resulting deformation of the free surface $z=\eta(x, y, t)$ is to be found as a part of the solution. It is assumed that the fluid is incompressible and the flow is irrotational. The former implies the existence of a velocity potential function $\phi(x, y, z, t)$ which fully describes the flow and the physical process. By definition, the fluid velocity vector can be expressed as $\vec{q}=\nabla \phi$ Thus, $\phi(x, y, z, t)$ must satisfy the Laplace's equation

$$
\nabla^{2} \phi(x, y, z, t)=0 \quad \text { where }(x, y, z) \in \Omega
$$

Also, $\phi(x, y, z, t)$ must satisfy the following kinematic and dynamic boundary conditions on the free surface and the solid boundary, respectively

$$
\begin{gathered}
\phi_{z}=\eta_{t}+\phi_{x} \eta_{x}+\phi_{y} \eta_{y} \text { on } z=\eta(x, y, t) \\
\phi_{z}=\zeta_{t}+\phi_{x} \zeta_{x}+\phi_{y} \zeta_{y} \text { on } z=-h+\zeta(x, y, t)
\end{gathered}
$$

and

$$
\phi_{t}=\frac{1}{2}(\nabla \phi)^{2}+g \eta=0 \quad \text { on } \quad z=\eta(x, y, t)
$$

where $g$ is the acceleration due to gravity. As described above, the initial conditions are given by

$$
\phi(x, y, z, 0)=\eta(x, y, 0)=\zeta(x, y, 0)=0
$$

The solution of (1)-(4) for a prescribed bed movement $\zeta(x, y, t)$ is inherently difficult owing to the nonlinear terms in the boundary conditions and the unknown location of the free surface a priori. The usual procedure for solving problems of this type is to circumvent these difficulties by substituting a linear approximation for the complete description of wave motion. In this approximation the nonlinear terms in the boundary conditions are omitted and the resulting equations are applied at the initial position of the boundaries.

\subsection{Linear Shallow Water Theory}

Various approximations can be considered for the full water-wave equations. One is the system of Boussinesq equations that retains nonlinearity and dispersion up to a certain order. Boussinesq model is used to study transient varying bottom problems. Fuhrman and Madsen [32] and Zhao et al. [33] presented a developed numerical model based on the highly accurate Boussinesq-type formulation subjected to exact expressions for the kinematic and dynamic free surface conditions. Their results show that the model was capable of treating the full life cycle of tsunami evolution, from the initial generation of bottom movements, to the subsequent propagation, and through the final run-up process. Reasonable computational efficiency has been demonstrated in their work, which made the model attractive for practical coastal engineering studies, where high dispersive and nonlinear accuracy is sought. Another one is the system of nonlinear shallow-water equations that retains nonlinearity but no dispersion. Solving this problem is a difficult task due to the nonlinearities and the priori unknown free surface. The simplest one is the system of linear shallow-water equations. The concept of shallow water is based on the smallness of the ratio between water depth and wave length. In the case of tsunamis propagating on the surface of deep oceans, one can consider that shallow-water theory is appropriate because the water depth (typically several kilometers) is much smaller than the wave length (typically several hundred kilometers), which is reasonable and usually true for most tsunamis triggered by submarine earthquakes, slumps and slides, [34,35]. Hence, the problem can be linearized by neglecting the nonlinear terms in the boundary conditions (2)-(4) and if the boundary conditions are applied on the nondeformed instead of the deformed boundary surfaces (i.e. $z=-h$ and on $z=0$ instead $z=-h+\zeta(x, y, t)$ of and $z=\eta(x, y, t))$.

The linearized problem in dimensional variables can be written as

$$
\nabla^{2} \phi(x, y, z, t)=0 \quad \text { where }(x, y, z) \in R^{2} \times[-h, 0]
$$

subjected to the following boundary conditions

$$
\begin{gathered}
\phi_{z}=\eta_{t} \text { on } \quad z=0 \\
\phi_{z}=\zeta_{t} \text { on } z=-h \\
\phi_{t}+g \eta=0 \text { on } z=0
\end{gathered}
$$

The linearized shallow water solution can be obtained by the Fourier-Laplace transforms.

\subsection{Solution of the Problem}

Our interest is focused on the resulting uplift of the free surface elevation $\eta(x, y, t)$. An analytical analysis is to examine and illustrate the generation and propagation of the tsunami for a given bed profile $\zeta(x, y, t)$. Mathematical modeling of waves generated by vertical and 
lateral displacements of ocean bottom using the combined Fourier-Laplace transforms of the Laplace equation analytically is the simplest way of studying tsunami development. All our studies took into account constant depth for which the Laplace and Fast Fourier Transform (FFT) methods could be applied. Equations (6)-(9) can be solved by using the method of integral transforms. We apply the Fourier transform in $(\mathrm{x}, \mathrm{y})$ given by

$$
\mathfrak{F}[f]=\hat{f}\left(k_{1}, k_{2}\right)=\int_{R^{2}} f(x, y) e^{-i\left(k_{1} x+k_{2} y\right)} d x d y
$$

with its inverse transform

$$
\mathfrak{F}^{-1}[\hat{f}]=f(x, y)=\frac{1}{(2 \pi)^{2}} \int_{R^{2}} \hat{f}\left(k_{1}, k_{2}\right) e^{i\left(k_{1} x+k_{2} y\right)} d k_{1} d k_{2}
$$

and the Laplace transform in time $\mathrm{t}$,

$$
£[g]=G(s)=\int_{0}^{\infty} g(t) e^{-s t} d t
$$

For the combined Fourier and Laplace transforms, the following notation is introduced

$$
\begin{aligned}
& \mathfrak{F}(\left(f(x, y, t)=\bar{F}\left(k_{1}, k_{2}, s\right)\right. \\
& \quad=\int_{R^{2}} e^{-i\left(k_{1} x+k_{2} y\right)} d x d y \int_{0}^{\infty} f(x, y, t) e^{-s t} d t
\end{aligned}
$$

Combining (7) and (9) yields the single free-surface condition

$$
\phi_{t t}(x, y, 0, t)+g \phi_{z}(x, y, 0, t)=0
$$

and the bottom condition (8) will be

$$
\phi_{z}(x, y,-h, t)=\zeta_{t}(x, y, t)
$$

The solution of the Laplace Equation (6) which satisfies the boundary conditions (10)-(11) can be obtained by using the Fourier-Laplace transforms method.

First, by applying the transforms method to the Laplace equation (6), gives

$$
\mathrm{F}\left\{£\left(\frac{\partial^{2} \phi}{\partial x^{2}}\right)\right\}+\mathrm{F}\left\{£\left(\frac{\partial^{2} \phi}{\partial y^{2}}\right)\right\}+\mathrm{F}\left\{£\left(\frac{\partial^{2} \phi}{\partial z^{2}}\right)\right\}=0
$$

By using the property $\mathfrak{F}\left\{\frac{d^{n} f}{\partial x^{n}}\right\}=(i k)^{n} \bar{F}(k)$,

will be

$$
\bar{\phi}_{t t}\left(k_{1}, k_{2}, z, s\right)-\left(k_{1}^{2}+k_{2}^{2}\right) \bar{\phi}\left(k_{1}, k_{2}, z, s\right)=0
$$

Second, by applying the transforms method to the boundary conditions (10)-(11) and the initial conditions (5), yields

$$
s^{2} \bar{\phi}\left(k_{1}, k_{2}, 0, s\right)+g \bar{\phi}_{z}\left(k_{1}, k_{2}, 0, s\right)=0
$$

and

$$
\bar{\phi}_{z}\left(k_{1}, k_{2},-h, s\right)=s \bar{\zeta}\left(k_{1}, k_{2}, s\right)
$$

The transformed free-surface elevation can be obtained from (9) as

$$
\bar{\eta}\left(k_{1}, k_{2}, s\right)=-\frac{s}{g} \bar{\phi}\left(k_{1}, k_{2}, 0, s\right)
$$

The general solution of (13) will be

$$
\begin{aligned}
\bar{\phi}\left(k_{1}, k_{2}, z, s\right) & =A\left(k_{1}, k_{2}, s\right) \cosh (k z) \\
& +B\left(k_{1}, k_{2}, s\right) \sinh (k z)
\end{aligned}
$$

where $k=\sqrt{k_{1}^{2}+k_{2}^{2}}$. The functions $A\left(k_{1}, k_{2}, s\right)$ and $B\left(k_{1}, k_{2}, s\right)$ can be found from the boundary conditions (14)-(15) as follows

For the bottom condition (at $z=-h$ ):

$$
\frac{\partial \bar{\phi}\left(k_{1}, k_{2},-h, s\right)}{\partial z}=-A k \sinh (k h)+B k \cosh (k h)
$$

Substituting from (18) into (15), yields

$$
-A k \sinh (k h)+B k \cosh (k h)=s \bar{\zeta}\left(k_{1}, k_{2}, s\right)
$$

For the free surface condition (at $z=0$ ):

$$
\frac{\partial \bar{\phi}\left(k_{1}, k_{2},-h, s\right)}{\partial z}=B k \text { and } \bar{\phi}\left(k_{1}, k_{2}, 0, s\right)=A
$$

Substituting from (20) into (14), gives

$$
A=\frac{-g k}{s^{2}} B
$$

Using (21), (19) can be written as

$$
B k \cosh (k h)\left[1+\frac{g k}{s^{2}} \tanh (k h)\right]=s \bar{\zeta}\left(k_{1}, k_{2}, s\right)
$$

From which,

$$
\begin{gathered}
A\left(k_{1}, k_{2}, s\right)=\frac{g s \bar{\zeta}\left(k_{1}, k_{2}, s\right)}{\cosh (k h)\left[s^{2}+g k \tanh (k h)\right]}, \\
B\left(k_{1}, k_{2}, s\right)=\frac{s^{3} \bar{\zeta}\left(k_{1}, k_{2}, s\right)}{k \cosh (k h)\left[s^{2}+g k \tanh (k h)\right]} .
\end{gathered}
$$

Substituting the expressions for the functions $A\left(k_{1}, k_{2}, s\right)$ and $B\left(k_{1}, k_{2}, s\right)$ in (17) yields,

$$
\begin{aligned}
\bar{\phi}\left(k_{1}, k_{2}, z, s\right)= & -\frac{g s \bar{\zeta}\left(k_{1}, k_{2}, s\right)}{\cosh (k h)\left(s^{2}+\omega^{2}\right)} \\
& \left(\cosh (k z)-\frac{s^{2}}{g k} \sinh (k z)\right)
\end{aligned}
$$


where $\omega=\sqrt{g k \tanh (k h)}$ is the circular frequency of the wave motion.

The free surface elevation $\bar{\eta}\left(k_{1}, k_{2}, s\right)$ can be obtained from (16) as

$$
\bar{\eta}\left(k_{1}, k_{2}, s\right)=\frac{s^{2} \bar{\zeta}\left(k_{1}, k_{2}, s\right)}{\cosh (k h)\left(s^{2}+\omega^{2}\right)}
$$

A solution for $\eta(x, y, t)$ can be evaluated for specified $\zeta(x, y, t)$ by computing approximately its transform $\bar{\zeta}\left(k_{1}, k_{2}, s\right)$ then substituting it into (24) and inverting $\bar{\eta}\left(k_{1}, k_{2}, s\right)$ to obtain $\eta(x, y, t)$ We are concerned now to evaluate $\eta(x, y, t)$ by transforming analytically the assumed source model then inverting $\bar{\eta}\left(k_{1}, k_{2}, s\right)$ using the inverse Laplace Transform to obtain $\bar{\eta}\left(k_{1}, k_{2}, t\right)$ which is further converted to $\eta(x, y, t)$ by using double inverse Fourier Transform.

The circular frequency $\omega$ describes the dispersion relation of tsunamis and implies phase velocity $c=\frac{\omega}{k}$ and group velocity $U=\frac{d \omega}{d k}$.

Hence, $c=\sqrt{\frac{g \tanh (k h)}{k}}$, and $U=\frac{1}{2} c\left(1+\frac{2 k h}{\sinh (2 k h)}\right)$.

Since, $k=\frac{2 \pi}{\lambda}$, hence as $k h \rightarrow 0$, both $c \rightarrow \sqrt{g h}$ and $U \rightarrow \sqrt{g h}$, which implies that the tsunami velocity $v_{t}=\sqrt{g h}$ for wavelengths $\lambda$ long compared to the water depth $h$. The above linearized solution is known as the shallow water solution. We considered three stages for the mechanism of the tsunami generation caused by submarine gravity mass flows, initiated by a rapid curvilinear down and uplift faulting with rise time $0 \leq t \leq t_{1}$, then propagating unilaterally in the positive $\mathrm{x}$-direction with time $t_{1} \leq t \leq t^{*}$, to a length $\mathrm{L}$ both with finite velocity $v$ to produce a depletion and an accumulation zones. The last stage represented by the time variation in the velocity of the accumulation slide (block slide) moving in the $\mathrm{x}$-direction with time $t^{*} \leq t \leq t_{\max }$ and deceleration $\alpha$, where $t_{\max }$ is the maximum time that the slide takes to stop with minimum deceleration $\alpha_{\text {min }}$. In the y-direction, the models propagate instantaneously. The set of physical parameters used in the problem are given in Table 1.

The first and second stages of the bed motion are
Table 1. Parameters used in the analytical solution of the problem.

\begin{tabular}{lcc}
\hline \multicolumn{1}{c}{ Parameters } & First stage & Second stage \\
\hline -Source width, W, km & 100 & 100 \\
$\begin{array}{l}\text {-Whole width in } 1^{\text {st }} \text { Stage and } \\
\text { Propagation length in } 2^{\text {nd }} \text { Stage, } \mathrm{km}\end{array}$ & $W^{\prime}=100$ & $L=150$ \\
-Water depth (uniform), h, km & 2 & 2 \\
$\begin{array}{l}\text {-Acceleration due to gravity, } \\
\text { g, km/sec }\end{array}$ & 0.0098 \\
$\begin{array}{l}\text {-Tsunami velocity, } \\
v_{t}=\sqrt{g h}, \mathrm{~km} / \mathrm{sec}\end{array}$ & 0.14 & 0.0098 \\
-Moving velocity, $v, \mathrm{~km} / \mathrm{sec}$ & 0.14 & 0.14 \\
$\begin{array}{l}\text {-Duration of the source process, } \\
\mathrm{t}, \text { min }\end{array}$ & $t_{1}=\frac{50}{v}=5.95$ & $t^{*}=\frac{200}{v}=23.8$ \\
\hline
\end{tabular}

shown in Figures 2 and 3, respectively, and given by:

1) First Stage: Curvilinear down and Uplift Faulting

$$
\begin{aligned}
& \zeta_{\text {down }}(x, y, t)= \\
& \left\{\begin{array}{l}
-\zeta_{0} \frac{v t}{2 W^{\prime}}\left(1+\cos \frac{\pi}{50} x\right)\left[1-\cos \frac{\pi}{100}(y+150)\right], \\
-50 \leq x \leq 50,-150 \leq y \leq-50, \\
-\zeta_{0} \frac{v t}{W^{\prime}}\left(1+\cos \frac{\pi}{50} x\right), \\
-50 \leq x \leq 50,-50 \leq y \leq 50, \\
-\zeta_{0} \frac{v t}{2 W^{\prime}}\left(1+\cos \frac{\pi}{50} x\right)\left[1+\cos \frac{\pi}{100}(y-50)\right], \\
-50 \leq x \leq 50,50 \leq y \leq 150 .
\end{array}\right. \\
& \zeta_{\text {up }}(x, y, t)= \\
& \zeta_{0} \frac{v t}{2 W^{\prime}}\left(1-\cos \frac{\pi}{50}(x-200)\right)\left[1-\cos \frac{\pi}{100}(y+150)\right], \\
& 200 \leq x \leq 300,-150 \leq y \leq-50, \\
& \left\{\zeta_{0} \frac{v t}{W^{\prime}}\left(1-\cos \frac{\pi}{50}(x-200)\right)\right. \\
& 200 \leq x \leq 300,-50 \leq y \leq 50, \\
& \begin{array}{l}
\zeta_{0} \frac{v t}{2 W^{\prime}}\left(1-\cos \frac{\pi}{50}(x-200)\right)\left[1+\cos \frac{\pi}{100}(y-50)\right], \\
200 \leq x \leq 300, \quad 50 \leq y \leq 150 .
\end{array}
\end{aligned}
$$

For these displacements, the bed rises during $0 \leq t \leq t_{1}$ to a maximum displacement $\zeta_{0}$ such that the volume of 


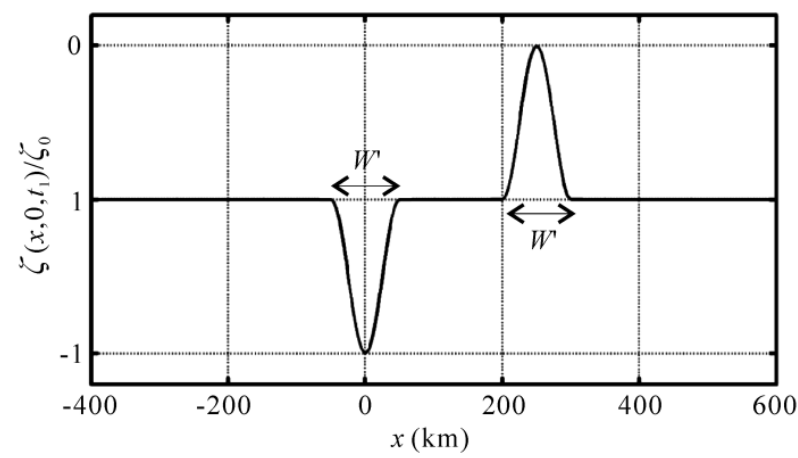

(a)

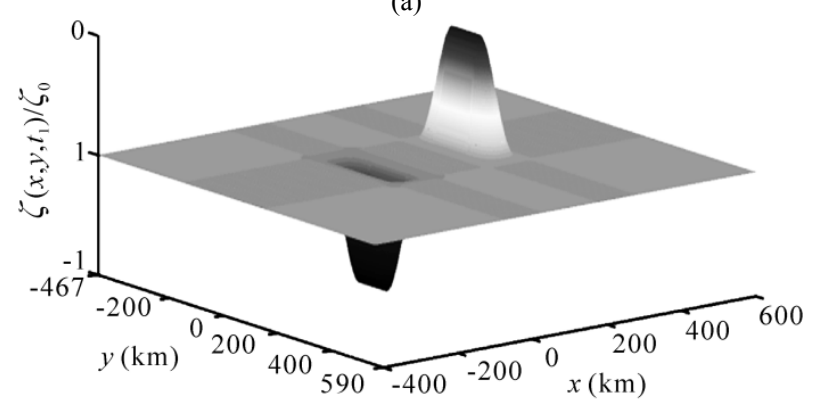

(b)

Figure 2. Normalized bed deformation represented by a rapid curvilinear down and uplift faulting at the end of stage one $\left(t_{1}=\frac{50}{v}\right)$ (a) Side view along the axis of symmetry at $y=0$ (b) Three- dimensional view.

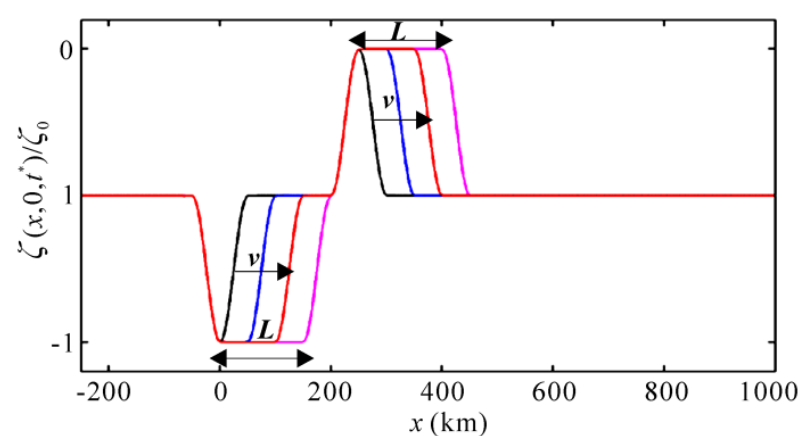

(a)

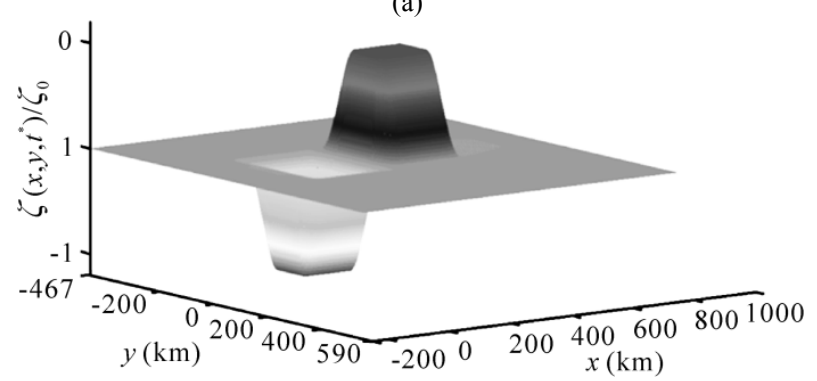

(b)

Figure 3. Normalized Bed deformation model represented by the accumulation and depletion zones at the end of stage two $\left(t^{*}=\frac{200}{v}\right)$ (a) Side view along the axis of symmetry at $y=0$ (b) Three-dimensional view. soil in the uplift increases linear with time and vise verse in the down faulting.

For $t \geq t_{1}$ the soil further propagates unilaterally in the positive $\mathrm{x}$-direction with velocity $v$ till it reaches the characteristic length $L=150 \mathrm{~km}$ at $t=t^{*}=200 / v$.

2) Second Stage: Curvilinear down and Uphill Slip-Fault (Slump and the Slide)

$$
\begin{aligned}
\zeta_{\text {down }}(x, y, t) & =\zeta_{\text {down }}(x, y, t)+\zeta_{\text {2down }}(x, y, t) \\
& +\zeta_{\text {3down }}(x, y, t)
\end{aligned}
$$

where,

$$
\begin{aligned}
& \zeta_{\text {Idown }}(x, y, t)= \\
& \left\{\begin{array}{l}
-\frac{\zeta_{0}}{4}\left(1+\cos \frac{\pi}{50} x\right)\left[1-\cos \frac{\pi}{100}(y+150)\right], \\
-50 \leq x \leq 0,-150 \leq y \leq-50, \\
-\frac{\zeta_{0}}{2}\left[1-\cos \frac{\pi}{100}(y+150)\right], \\
0 \leq x \leq\left(t-t_{1}\right) v,-150 \leq y \leq-50, \\
-\frac{\zeta_{0}}{4}\left(1+\cos \frac{\pi}{50}\left(x-\left(t-t_{1}\right) v\right)\right)\left[1-\cos \frac{\pi}{100}(y+150)\right], \\
\left(t-t_{1}\right) v \leq x \leq\left(t-t_{1}\right) v+50,-150 \leq y \leq-50 .
\end{array}\right.
\end{aligned}
$$$$
\zeta_{\text {2down }}(x, y, t)=
$$$$
-\frac{\zeta_{0}}{2}\left(1+\cos \frac{\pi}{50} x\right) \text {, }
$$$$
-50 \leq x \leq 0,-50 \leq y \leq 50,
$$$$
-\zeta_{0}
$$$$
0 \leq x \leq\left(t-t_{1}\right) v, \quad-50 \leq y \leq 50,
$$$$
-\frac{\zeta_{0}}{2}\left[1+\cos \frac{\pi}{50}\left(x-\left(t-t_{1}\right) v\right)\right] \text {, }
$$$$
\left(t-t_{1}\right) v \leq x \leq\left(t-t_{1}\right) v+50,-50 \leq y \leq 50 \text {. }
$$

$\zeta_{\text {3down }}(x, y, t)=$

$\left\{\begin{array}{l}-\frac{\zeta_{0}}{4}\left(1+\cos \frac{\pi}{50} x\right)\left[1+\cos \frac{\pi}{100}(y-50)\right] \\ -50 \leq x \leq 0,50 \leq y \leq 150 \\ -\frac{\zeta_{0}}{2}\left[1+\cos \frac{\pi}{100}(y-50)\right] \\ 0 \leq x \leq\left(t-t_{1}\right) v, 50 \leq y \leq 150 \\ -\frac{\zeta_{0}}{4}\left[1+\cos \frac{\pi}{50}\left(x-\left(t-t_{1}\right) v\right)\right]\left[1+\cos \frac{\pi}{100}(y-50)\right] \\ \left(t-t_{1}\right) v \leq x \leq\left(t-t_{1}\right) v+50,50 \leq y \leq 150 .\end{array}\right.$

$\zeta_{\text {up }}(x, y, t)=\zeta_{\text {lup }}(x, y, t)+\zeta_{\text {2up }}(x, y, t)+\zeta_{\text {3up }}(x, y, t)$ 
By referring to Figure 3, $\zeta_{\text {lup }}(x, y, t), \zeta_{\text {2up }}(x, y, t)$ and $\zeta_{\text {3up }}(x, y, t)$ can be expressed in the same manner as $\zeta_{\text {1down }}(x, y, t) \quad \zeta_{\text {2down }}(x, y, t)$ and $\zeta_{\text {3down }}(x, y, t)$.

The kinematic realistic tsunami source model shown in Figure 3 is initiated by a rapid curvilinear down and uplift faulting (First stage) which then spreads unilaterally with constant velocity $v$ causing a depletion and accumulation zones. The final down lift of the depression zone and final uplift of the accumulation zone are assumed to have the same amplitude $\zeta_{0}$. We assume the spreading velocity $v$ of the slump and the slide deformation in Figure 3 the same as the tsunami wave velocity $v_{t}=\sqrt{g h}$ as the largest amplification of the tsunami amplitude occurs when $v=v_{t}$ due to wave focusing $[6,36]$. The slide and the slump are assumed to have constant width $\mathrm{W}$.

The spreading is unilateral in the $\mathrm{x}$-direction as shown in Figure 3. The vertical displacement, $\zeta_{0}$, is negative (downwards) in zones of depletion, and positive (upwards) in zones of accumulation. All cases are characterized by sliding motion in one direction, without loss of generality coinciding with the $\mathrm{x}$-axis, and tsunami propagating in the $x-y$ plane.

Figure 4 shows vertical cross-sections (through y $=0$ ) of the mathematical models of the stationary submarine slump and the moving slide and their schematic representation of the physical process that we considered in this study, as those evolve for time $t \geq t^{*}$. The block slide starts moving in the positive $\mathrm{x}$-direction at time $t=t^{*}$ and stops moving at distance $L^{\prime}=150 \mathrm{~km}$ while the downhill slide becomes stationary. We discuss the tsunami generation for two cases of the movement of the block slide. First, the limiting case in which the block slide moves with constant velocity $v$ and stops after distance $L^{\prime}$ with infinite deceleration (sudden stop) at time $t_{\min }=t^{*}+L^{\prime} / v$. Second, the general case in which the block slide moves in time $t_{2} \geq t^{*}$. With constant velocity and then with constant deceleration such that it stops softly after traveling the same distance $L^{\prime}$ in time $t_{3}$ which depends on the deceleration $\alpha$ and the choice of time $t_{2}$. The variation of the "block slide" we considered could be used to represent the motion of the collapsed blocks at the Blake Escarpment, east of Florida. (see Figure 7 in Dillon et al. [37]) and the block slide at the base of Middle Canyon along the Beringian Margin in Alaska (see Figure 8 in Carlson et al. [38]) and the Sur submarine landslide originated on the continental slope west of Point Sur, central California(see Figure 1 in Gutmacher and Normark [39]). So, the evidence of a huge historical tsunami needs for investigating the possibility of future tsunami generating by submarine land- slides.

The velocity $v(t)$ in this case can be defined as

$$
v(t)=\left\{\begin{array}{cc}
v & t^{*} \leq t \leq t_{2} \\
v-\left(t-t_{2}\right) \alpha & t_{2} \leq t \leq t_{3}
\end{array}\right.
$$

where $v=v_{t}=0.14 \mathrm{~km} / \mathrm{sec}$ and $\alpha$ is the deceleration of the moving block slide. We need to determine the time $t_{3}$ that the slide takes to reach the final distance $L^{\prime}$ and

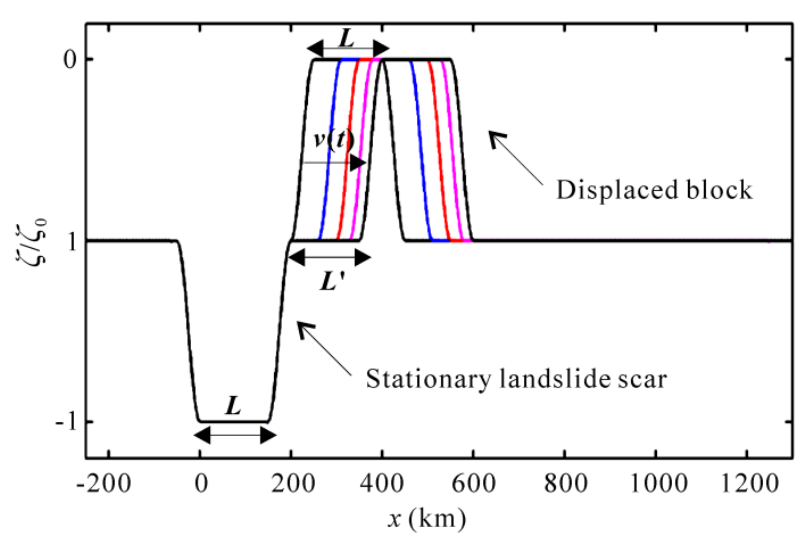

(a)

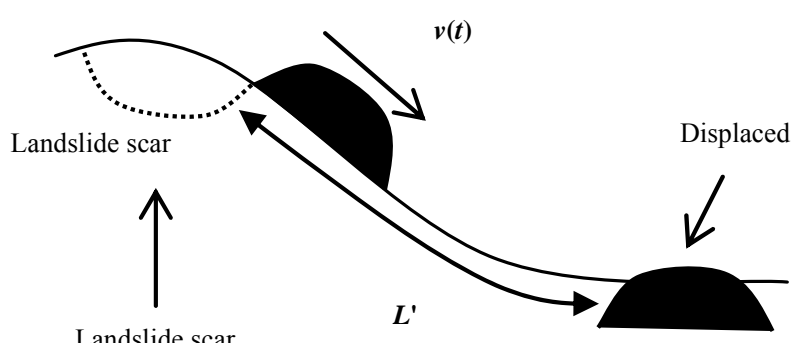

(b)

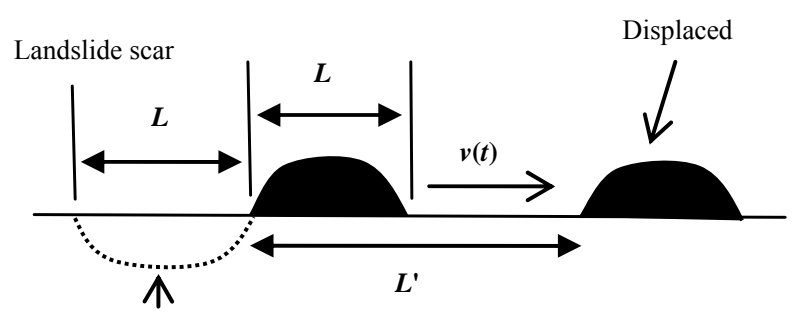

(c)

Figure 4. A schematic representation of a landslide (bottom) travelling a significant distance $L$ downhill creating a "scar" and a moving uphill displaced block slide stopping at the characteristic length $L^{\prime}$. (a) Case 1: Mathematical model of the stationary slump and the moving submarine slide; (b) Case 2: Physical process of the displaced block moving with a variable slide velocity $v(t)$; (c) Case 3 : Schematic representation of the used model. 
the corresponding deceleration $\alpha$. This can be done by the following steps:

1) Choosing time $t_{2}$ as $t^{*} \leq t_{2} \leq t^{*}+\left(L^{\prime} / v\right)$ in which the slide moves with constant velocity $v$ where $t^{*}=t_{1}+L / v$ and $t_{1}=50 / v$.

2) Getting the corresponding distance

$$
L^{*}=\left(t_{2}-t^{*}\right) v
$$

3) Evaluating the remaining distance

$$
L^{* *}=L^{\prime}-\left(t_{2}-t^{*}\right) v .
$$

Substituting $L^{* *} \mathrm{n}$ the equation

$$
L^{* *}=\left(t_{3}-t_{2}\right) v-\frac{1}{2}\left(t_{3}-t_{2}\right)^{2}
$$

When the block slide stops moving, then

$$
v(t)=v-\left(t_{3}-t_{2}\right)\left(t_{3}-t_{2}\right)=0
$$

Eliminating $\alpha$ from (30) and (31), we get a relation between $t_{3}$ and $t_{2}$ which further on substituting in (31), we obtain the deceleration $\alpha$.

For $t_{2} \leq t \leq t_{3}$, the block slide moves with velocity

\begin{tabular}{|c|c|c|}
\hline Time $t_{2}$ (minutes) & Time $t_{3}$ (minutes) & Deceleration $\alpha$ \\
\hline$t^{*}=23.80$ & 59.51 & $6.53 \times 10^{-5}$ \\
\hline$t^{*}+0.1(L / v)=25.58$ & 57.72 & $7.25 \times 10^{-5}$ \\
\hline$t^{*}+0.2(L ' / v)=27.37$ & 55.95 & $8.16 \times 10^{-5}$ \\
\hline$t^{*}+0.3(L ' / v)=29.37$ & 54.15 & $9.33 \times 10^{-5}$ \\
\hline$t^{*}+0.4(L ' / v)=30.94$ & 52.36 & $1.08 \times 10^{-4}$ \\
\hline$t^{*}+0.5(L / v)=32.72$ & 50.57 & $1.30 \times 10^{-4}$ \\
\hline$t^{*}+0.6(L ' / v)=34.51$ & 48.79 & $1.63 \times 10^{-4}$ \\
\hline$t^{*}+0.7(L ' / v)=36.30$ & 47.01 & $2.17 \times 10^{-4}$ \\
\hline$t^{*}+0.8(L / \mathrm{v})=38.08$ & 45.22 & $3.26 \times 10^{-4}$ \\
\hline$t^{*}+0.9(L ' v)=39.87$ & 43.44 & $6.53 \times 10^{-4}$ \\
\hline$t^{*}+\left(L^{\prime} / v\right)=41.65$ & 41.65 & infinity \\
\hline
\end{tabular}
$v(t)=v-\left(t-t_{2}\right) \alpha$. Table 2 represents the different

Table 2. Values of $t_{2}$ and the corresponding calculated values of $t_{3}$ and $\alpha$. values of $t_{2}$ and the corresponding calculated values of $t_{3}$ and $\alpha$.

Figure 5 illustrates the position of the slides in the third stage for different choice of deceleration $\alpha$. In this stage, $\alpha_{\min }$ is the minimum deceleration required such that the slide stops after traveling distance $L^{\prime}$.

In this case $t_{2}=t^{*}$ and $t_{3}=t_{\max }=t^{*}+\left(2 L^{\prime} / v\right)=$ $59.51 \mathrm{~min}$. For any other $\alpha>\alpha_{\text {min }}$, the slide moves with constant velocity with time $t^{*} \leq t \leq t_{2}$ and with deceleration $\alpha$ until it stops at time $t_{3}$ which is less than $t_{\max }$.

So, the stationary landslide scar for $t^{*} \leq t$ and the movable block slide with variable velocity $v(t)$ for $t^{*} \leq t_{2} \leq t^{*}+\left(L^{\prime} / v\right)$ and $t^{*}+\left(L^{\prime} / v\right) \leq t_{3} \leq t^{*}+\left(2 L^{\prime} / v\right)$ can be expressed respectively as

$$
\begin{aligned}
\zeta_{\text {stat.landslide }}\left(x, y, t^{*}\right) & =\zeta_{1}\left(x, y, t^{*}\right)+\zeta_{2}\left(x, y, t^{*}\right) \\
& +\zeta_{3}\left(x, y, t^{*}\right) \\
\zeta_{\text {block slide }}(x, y, t) & =\zeta_{1}(x, y, t)+\zeta_{2}(x, y, t) \\
& +\zeta_{3}(x, y, t)
\end{aligned}
$$

$\zeta_{\text {stat.landslide }}\left(x, y, t^{*}\right)$ is the same as (27) except the time parameter $t$ will be substituted by $t^{*}$.

$$
\text { For } \zeta_{\text {block slide }}(x, y, t) \text {, let } \eta(\mathrm{x}, 0, \mathrm{t})
$$

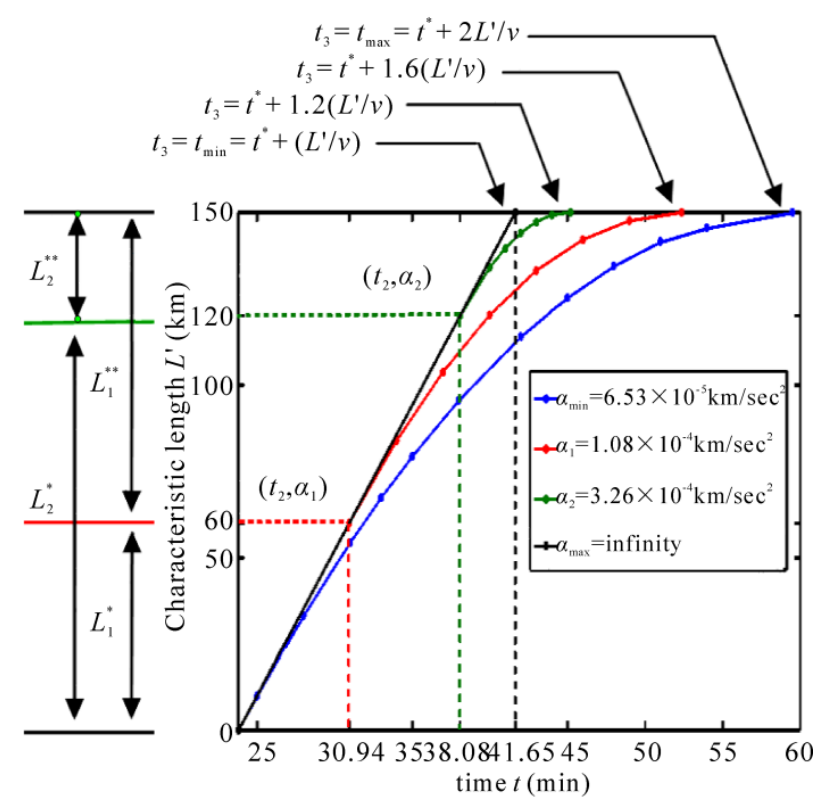

Figure 5. Slide block position against the instants of times $t^{*} \leq t_{2} \leq t^{*}+\left(L^{\prime} / v\right)$ and $t_{\text {min }} \leq t_{3} \leq t_{\text {max }}$. 


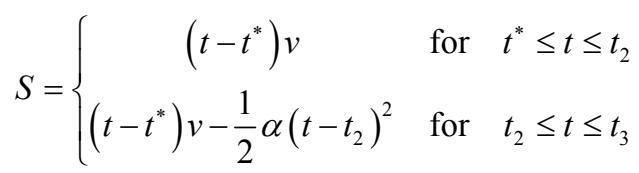

be the distance the slide moves during stage three, hence

$$
\begin{aligned}
& \zeta_{1}(x, y, t)= \\
& \left\{\begin{array}{l}
\frac{\zeta_{0}}{4}\left(1-\cos \frac{\pi}{50}(x-(200+S))\right)\left[1-\cos \frac{\pi}{100}(y+150)\right], \\
200+S \leq x \leq 250+S,-150 \leq y \leq-50, \\
\frac{\zeta_{0}}{2}\left[1-\cos \frac{\pi}{100}(y+150)\right], \\
250+S \leq x \leq 250+S+L,-150 \leq y \leq-50, \\
\frac{\zeta_{0}}{4}\left[1+\cos \frac{\pi}{50}(x-(250+S+L))\right]\left[1-\cos \frac{\pi}{100}(y+150)\right], \\
250+S+L \leq x \leq 300+S+L,-150 \leq y \leq-50 .
\end{array}\right. \\
& \zeta_{2}(x, y, t)= \\
& \left\{\begin{array}{l}
\frac{\zeta_{0}}{2}\left(1-\cos \frac{\pi}{50}(x-(200+S))\right), \\
200+S \leq x \leq 250+S,-50 \leq y \leq 50, \\
\zeta_{0}, \\
250+S \leq x \leq 250+S,-50 \leq y \leq 50, \\
\frac{\zeta_{0}}{2}\left[1+\cos \frac{\pi}{50}(x-(250+S+L))\right], \\
250+S+L \leq x \leq 300+S+L,-50 \leq y \leq 50 .
\end{array}\right.
\end{aligned}
$$

$$
\begin{aligned}
& \zeta_{3}(x, y, t)= \\
& \left\{\begin{array}{l}
\frac{\zeta_{0}}{4}\left(1-\cos \frac{\pi}{50}(x-(200+S))\right)\left[1+\cos \frac{\pi}{100}(y-150)\right], \\
200+S \leq x \leq 250+S, 50 \leq y \leq 150, \\
\frac{\zeta_{0}}{2}\left[1+\cos \frac{\pi}{100}(y-150)\right], \\
250+S \leq x \leq 250+S+L, 50 \leq y \leq 150, \\
\frac{\zeta_{0}}{4}\left[1+\cos \frac{\pi}{50}(x-(250+S+L))\right]\left[1+\cos \frac{\pi}{100}(y-150)\right], \\
250+S+L \leq x \leq 300+S+L, 50 \leq y \leq 150 .
\end{array}\right.
\end{aligned}
$$

Laplace and Fourier transforms can now be applied to the bed motion described by (25)-(28) and (32)-(33). First, beginning with the down and uplift faulting (25) and (26) for $0 \leq t \leq t_{1}$ where $t_{1}=\frac{50}{v}$, and

$$
\begin{aligned}
\mathfrak{F}(£(\zeta(x, y, t) & =\bar{\zeta}\left(k_{1}, k_{2}, s\right) \\
& =\int_{-\infty}^{\infty} e^{-i\left(k_{1} x+k_{2} y\right)} d x d y \int_{0}^{\infty} \zeta(x, y, t) e^{-s t} d t
\end{aligned}
$$

The limits of the above integration are apparent from (25)-(26).

Substituting the results of the integration (34) for $\zeta_{\text {down }}$ and $\zeta_{\text {up }}$ into (24). The free surface elevation $\bar{\eta}\left(k_{1}, k_{2}, t\right)$ can be evaluated by using the inverse Laplace transforms of $\bar{\eta}\left(k_{1}, k_{2}, s\right)$. From which,

$$
\begin{aligned}
& \bar{\eta}\left(k_{1}, k_{2}, t\right)=\frac{\sin \omega t}{\omega \cosh (k h)} \times \frac{\zeta_{0} v}{2 W^{\prime}}\left[\left[\frac{1}{1-\left(\frac{50}{\pi} k_{1}\right)^{2}}\left[i k_{1}\left(\frac{50}{\pi}\right)^{2}\left(e^{-i 300 k_{1}}-e^{i 200 k_{1}}\right)\right]\right]\right. \\
& \times\left[\begin{array}{l}
\left.\left[\frac{e^{i 150 k_{2}}-e^{i 50 k_{2}}}{i k_{2}}-\frac{1}{1-\left(\frac{100}{\pi} k_{2}\right)^{2}}\left[i k_{2}\left(\frac{100}{\pi}\right)^{2}\left(e^{i 50 k_{2}}+e^{i 150 k_{2}}\right)\right]\right]+\frac{4 \sin \left(50 k_{2}\right)}{k_{2}}\right] \\
+\left[\frac{e^{-i 50 k_{2}}-e^{-i 150 k_{2}}}{i k_{2}}+\frac{1}{1-\left(\frac{100}{\pi} k_{2}\right)^{2}}\left[i k_{2}\left(\frac{100}{\pi}\right)^{2}\left(e^{-i 150 k_{2}}+e^{-i 50 k_{2}}\right)\right]\right]
\end{array}\right]
\end{aligned}
$$


In case of $t \geq t_{1}, \bar{\eta}\left(k_{1}, k_{2}, t\right)$ will have the same expression except in the convolution step, the integral becomes $\int_{0}^{t} \cos \omega \tau d \tau=\frac{\sin \omega t}{\omega}-\frac{\sin \omega\left(t-t_{1}\right)}{\omega}$ instead of $\int_{0}^{t} \cos \omega \tau d \tau=\frac{\sin \omega t}{\omega}$.

Finally, $\eta(x, y, t)$ is evaluated using the double inverse Fourier transform of $\bar{\eta}\left(k_{1}, k_{2}, t\right)$

$$
\eta(x, y, t)=\frac{1}{(2 \pi)^{2}} \int_{-\infty}^{\infty} e^{i k_{2} y}\left[\int_{-\infty}^{\infty} e^{i k_{1} x} \bar{\eta}\left(k_{1}, k_{2}, t\right) d k_{1}\right] d k_{2}
$$

This inversion is computed by using the FFT. The inverse FFT is a fast algorithm for efficient implementation of the Inverse Discrete Fourier Transform (IDFT) given by

$$
\begin{aligned}
& f(m, n)=\frac{1}{M N} \sum_{p=0}^{M-1} \sum_{q=0}^{N-1} F(p, q) e^{i\left(\frac{2 \pi}{M}\right) p m} e^{i\left(\frac{2 \pi}{N}\right) q n}, \\
& p=0,1, \ldots, M-1, \quad q=0,1, \ldots N-1,
\end{aligned}
$$

where $f(m, n)$ is the resulting function of the two spatial variables $m$ and $n$, corresponding to $x$ and $y$, from the frequency domain function $F(p, q)$ with frequency variables $p$ and $q$, corresponding to $k_{1}$ and $k_{2}$. This inversion is done efficiently by using the Matlab FFT algorithm.

Using the same steps, $\bar{\eta}\left(k_{1}, k_{2}, t\right)$ is evaluated by applying the Laplace and Fourier transforms to the bed motion described by (27) and (28), then substituting into (24) and then inverting $\bar{\eta}\left(k_{1}, k_{2}, s\right)$ using the inverse Laplace transform to obtain $\bar{\eta}\left(k_{1}, k_{2}, t\right)$. This is verified for $t_{1} \leq t \leq t^{*}$ where $t^{*}=\frac{200}{v}$ as

$$
\bar{\eta}\left(k_{1}, k_{2}, t\right)=\bar{\eta}_{\text {down }}\left(k_{1}, k_{2}, t\right)+\bar{\eta}_{\text {up }}\left(k_{1}, k_{2}, t\right)
$$

where,

$$
\begin{aligned}
\bar{\eta}_{\text {down }}\left(k_{1}, k_{2}, t\right) & =\bar{\eta}_{\text {down }}\left(k_{1}, k_{2}, t\right)+\bar{\eta}_{\text {2down }}\left(k_{1}, k_{2}, t\right) \\
& +\bar{\eta}_{3 \text { down }}\left(k_{1}, k_{2}, t\right)
\end{aligned}
$$

then

and

$$
\begin{aligned}
\bar{\eta}_{\text {up }}\left(k_{1}, k_{2}, t\right) & =\bar{\eta}_{\text {lup }}\left(k_{1}, k_{2}, t\right)+\bar{\eta}_{\text {2up }}\left(k_{1}, k_{2}, t\right) \\
& +\bar{\eta}_{\text {3up }}\left(k_{1}, k_{2}, t\right)
\end{aligned}
$$

then

$$
\begin{aligned}
& \left.\bar{\eta}_{\text {down }}\left(k_{1}, k_{2}, t\right)=\left[\begin{array}{l}
{\left[\frac{-\zeta_{0}}{4 \cosh (k h)}\right] \times\left[\frac{e^{i 150 k_{2}}-e^{i 50 k_{2}}}{i k_{2}}-\frac{1}{1-\left(\frac{100}{\pi} k_{2}\right)^{2}}\left[i k_{2}\left(\frac{100}{\pi}\right)^{2}\left(e^{i 50 k_{2}}+e^{i 150 k_{2}}\right)\right]\right.} \\
+\left[\frac{-\zeta_{0}}{\cosh (k h)}\right]\left[\frac{\sin \left(50 k_{2}\right)}{k_{2}}\right] \\
+\left[\frac{-\zeta_{0}}{4 \cosh (k h)}\right] \times\left[\frac{e^{-i 50 k_{2}}-e^{-i 150 k_{2}}}{i k_{2}}-\frac{1}{1-\left(\frac{100}{\pi} k_{2}\right)^{2}}\left[i k_{2}\left(\frac{100}{\pi}\right)^{2}\left(e^{-i 150 k_{2}}+e^{-i 50 k_{2}}\right)\right]\right.
\end{array}\right]\right]
\end{aligned}
$$

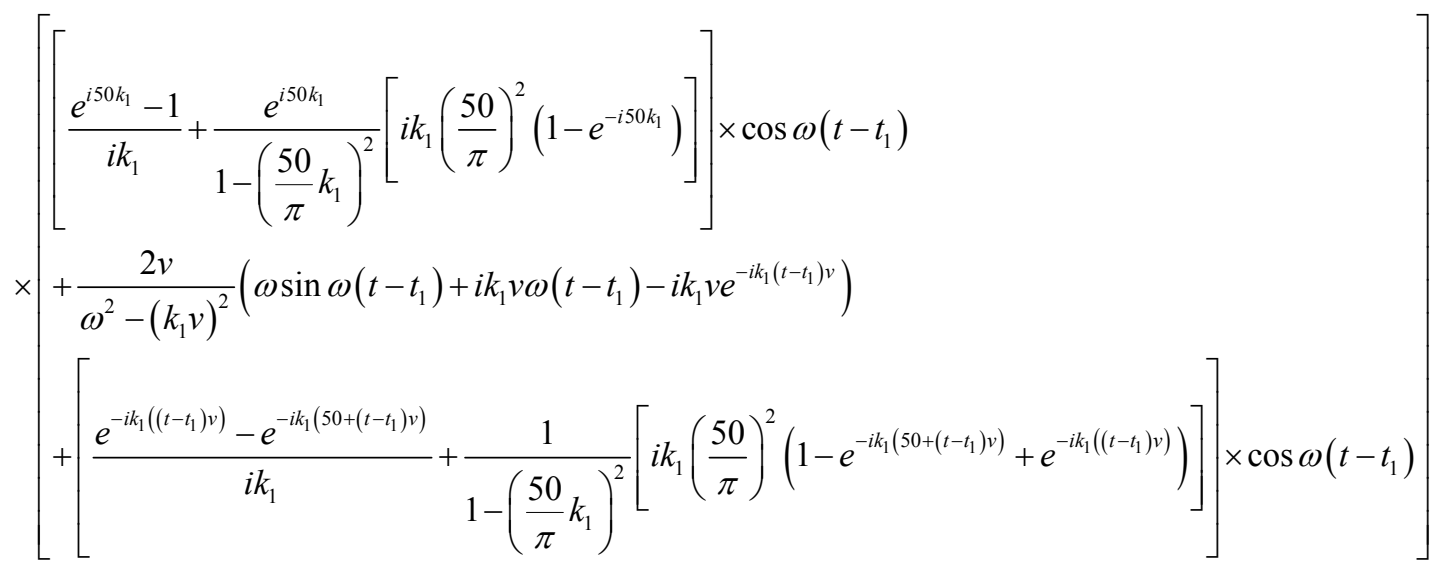




$$
\begin{aligned}
& \bar{\eta}_{\text {up }}\left(k_{1}, k_{2}, t\right)=\left[\begin{array}{l}
{\left[\frac{\zeta_{0}}{4 \cosh (k h)}\right] \times\left[\frac{e^{i 150 k_{2}}-e^{i 50 k_{2}}}{i k_{2}}-\frac{1}{1-\left(\frac{100}{\pi} k_{2}\right)^{2}} \times\left[i k_{2}\left(\frac{100}{\pi}\right)^{2}\left(e^{i 50 k_{2}}+e^{i 150 k_{2}}\right)\right]\right.} \\
+\left[\frac{\zeta_{0}}{\cosh (k h)}\right]\left[\frac{\sin \left(50 k_{2}\right)}{k_{2}}\right] \\
+\left[\frac{\zeta_{0}}{4 \cosh (k h)}\right] \times\left[\frac{e^{-i 50 k_{2}}-e^{-i l 50 k_{2}}}{i k_{2}}+\frac{1}{1-\left(\frac{100}{\pi} k_{2}\right)^{2}} \times\left[i k_{2}\left(\frac{100}{\pi}\right)^{2}\left(e^{-i 150 k_{2}}+e^{-i 50 k_{2}}\right)\right]\right.
\end{array}\right] \\
& \times\left[\begin{array}{l}
{\left[\begin{array}{l}
\frac{e^{-i 200 k_{1}}-e^{-i 250 k_{1}}}{i k_{1}}-\frac{1}{1-\left(\frac{50}{\pi} k_{1}\right)^{2}} \times\left[i k_{1}\left(\frac{50}{\pi}\right)^{2}\left(e^{-i 250 k_{1}}+e^{-i 200 k_{1}}\right)\right] \times \cos \omega\left(t-t_{1}\right) \\
+\frac{2 v e^{-i 250 k_{1}}}{\omega^{2}-\left(k_{1} v\right)^{2}}\left(\omega \sin \omega\left(t-t_{1}\right)+i k_{1} v \cos \omega\left(t-t_{1}\right)-i k_{1} v e^{-i k_{1}\left(t-t_{1}\right) v}\right) \\
{\left[\begin{array}{l}
{\left[\frac{e^{-i k_{1}\left(250+\left(t-t_{1}\right) v\right)}-e^{-i k_{1}\left(300+\left(t-t_{1}\right) v\right)}}{i k_{1}}-\frac{1}{1-\left(\frac{50}{\pi} k_{1}\right)^{2}} \times\left[i k_{1}\left(\frac{50}{\pi}\right)^{2}\left(e^{-i k_{1}\left(300+\left(t-t_{1}\right) v\right)}+e^{-i k_{1}\left(250+\left(t-t_{1}\right) v\right)}\right)\right]\right.}
\end{array}\right]} \\
\times \cos \omega\left(t-t_{1}\right)
\end{array}\right]}
\end{array}\right.
\end{aligned}
$$

Substituting $\bar{\eta}_{\text {down }}\left(k_{1}, k_{2}, t\right)$ and $\bar{\eta}_{\text {up }}\left(k_{1}, k_{2}, t\right)$ into (37) gives $\bar{\eta}\left(k_{1}, k_{2}, t\right)$ for $t_{1} \leq t \leq t^{*}$. For the case $t \geq t^{*}, \bar{\eta}\left(k_{1}, k_{2}, t\right)$ will have the same expression as (37) except for the term resulting from the convolution theorem, i.e.

$$
\begin{aligned}
& \int_{\left(t-t_{1}\right)-t^{*}}^{t} \cos \omega \tau e^{-i k_{1}\left(\left(t-t_{1}\right) v-\tau\right)} d \tau=\frac{1}{\omega^{2}-\left(k_{1} v\right)^{2}} \\
& {\left[\begin{array}{l}
\omega \sin \omega\left(t-t_{1}\right)+i k_{1} v \cos \omega\left(t-t_{1}\right)-e^{-i k_{1} v t^{*}} \\
\times\left(\omega \sin \omega\left(\left(t-t_{1}\right)-t^{*}\right)+i k_{1} v \cos \omega\left(\left(t-t_{1}\right)-t^{*}\right)\right)
\end{array}\right],}
\end{aligned}
$$

instead of

$$
\begin{aligned}
& \int_{\left(t-t_{1}\right)}^{t} \cos \omega \tau e^{-i k_{1}\left(t-t_{1}\right) v} d \tau=\frac{1}{\omega^{2}-\left(k_{1} v\right)^{2}} \\
& \left(\omega \sin \omega\left(t-t_{1}\right)+i k_{1} v \cos \omega\left(t-t_{1}\right)-i k_{1} v e^{-i k_{1} v\left(t-t_{1}\right) v}\right)
\end{aligned} .
$$

So, $\eta(x, y, t)$ is computed using inverse FFT of $\bar{\eta}\left(k_{1}, k_{2}, t\right)$.

Finally, $\bar{\eta}_{\text {block slide }}\left(k_{1}, k_{2}, t\right)$ is evaluated by applying the Laplace and Fourier transforms to the block slide motion described by (33), then substituting into (24) and then inverting $\bar{\eta}_{\text {block slide }}\left(k_{1}, k_{2}, s\right)$ using the inverse Laplace transform to obtain $\bar{\eta}_{\text {block slide }}\left(k_{1}, k_{2}, t\right)$. This is verified for $t^{*} \leq t_{2} \leq t^{*}+\left(L^{\prime} / v\right)$ and $t^{*}+\left(L^{\prime} / v\right) \leq t_{3}$ $\leq t^{*}+\left(2 L^{\prime} / v\right)$. Then,

$$
\begin{aligned}
\bar{\eta}_{\text {block slide }}\left(k_{1}, k_{2}, t\right) & =\bar{\eta}_{1}\left(k_{1}, k_{2}, t\right)+\bar{\eta}_{2}\left(k_{1}, k_{2}, t\right) \\
& +\bar{\eta}_{3}\left(k_{1}, k_{2}, t\right)
\end{aligned}
$$

Then,

Finally, $\eta(x, y, t)$ is computed using inverse FFT of $\bar{\eta}\left(k_{1}, k_{2}, t\right)$.

We investigated mathematically the water wave motion in the near and far-field by considering a kinematic mechanism of the sea floor faulting represented in sequence by a down and uplift motion with time followed by unilateral spreading in $\mathrm{x}$-direction, both with constant velocity $v$, then a deceleration movement of a block slide in the direction of propagation. Clearly, from the mathematical derivation done above, $\eta(x, y, t)$ depends continuously on the source $\zeta(x, y, t)$. Hence, from the mathematical point of view, this problem is said to be well-posed for modeling the physical processes of the tsunami wave. 


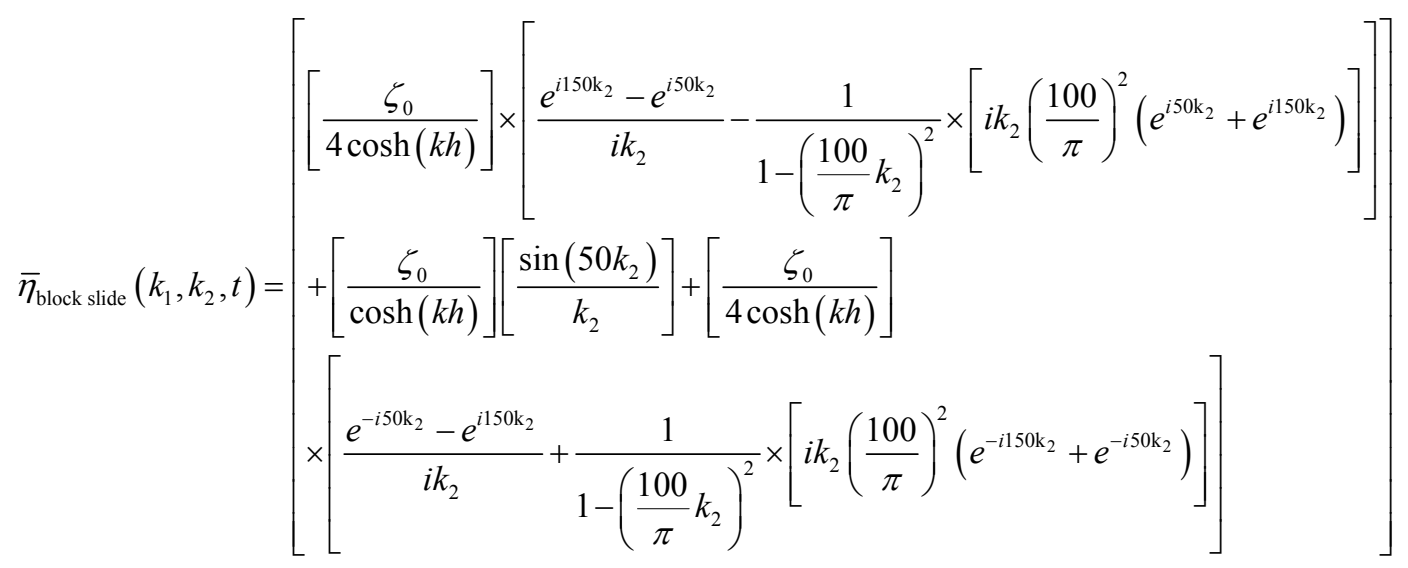

$$
\begin{aligned}
& \times\left[\begin{array}{l}
{\left[\begin{array}{l}
\frac{e^{-i(200+S) k_{1}}-e^{-i(250+S) k_{1}}}{i k_{1}}+\frac{1}{1-\left(\frac{50}{\pi} k_{1}\right)^{2}}-\left[i k_{1}\left(\frac{50}{\pi}\right)^{2}\left(e^{-i(250+S) k_{1}}+e^{-i(200+S) k_{1}}\right)\right] \\
\times \cos w\left(t_{3}-t^{*}\right) \\
+\frac{2 v e^{-i(250+S) k_{1}}}{w^{2}-\left(k_{1} v\right)^{2}}\left(w \sin w t_{3}+i k_{1} v \cos w t_{3}-e^{-i k_{1} L}\left(w \sin w\left[t_{3}-t^{*}\right]+i k_{1} v \cos w\left[t_{3}-t^{*}\right]\right)\right) \\
{\left[\begin{array}{l}
i k_{1} \\
+\left[\frac{e^{-i k_{1}(250+S+L)}-e^{-i k_{1}(300+S+L)}}{1-\left(\frac{50}{\pi} k_{1}\right)^{2}}\left[i k_{1}\left(\frac{50}{\pi}\right)^{2}\left(e^{-i k_{1}(300+S+L)}-e^{-i k_{1}(250+S+L)}\right)\right]\right.
\end{array}\right]} \\
\times \cos w\left(t_{3}-t^{*}\right)
\end{array}\right]}
\end{array}\right]
\end{aligned}
$$

\section{Results and Discussion}

We are interested in illustrating the nature of the tsunami build up and propagation during and after the movement process of a variable curvilinear block shape sliding. In this section three cases are studied. We first examine the generation process of tsunami waveform resulting from the unilateral spreading of the down and uplift slip faulting in the direction of propagation with constant velocity $v$. We assume the spreading velocity of the ocean floor up and down lift to be equal to the tsunami wave velocity $v_{t}=\sqrt{g h}=0.14 \mathrm{~km} / \mathrm{sec}$ as it has been verified in $[6,36]$ that the largest wave amplitude occurs when $v=v_{t}$ due to wave focusing.

\subsection{Tsunami Generation caused by Submarine Slump and Slide-Evolution in Time}

We assume the waveform initiated by a rapid movement of the bed deformation of the down and uplift source shown in Figure 2. Figure 6 shows the tsunami gener- ated waveforms during the second stage at time evolution $t=0.4 t^{*}, 0.6 t^{*}, 0.8 t^{*}, t^{*}$. at constant water depth $h=$ $2 \mathrm{~km}$. It is seen how the amplitude of the wave builds up progressively as $t$ increases where more water is lifted below the leading wave depending on its variation in time and the space in the source area. The wave will be focusing and the amplification may occur above the spreading edge of the slip. This amplification occurs above the source progressively as the source evolves by adding uplifted fluid to the fluid displaced previously by uplifts of preceding source segments. This explains why the amplification is larger for wider area of uplift source than for small source area. It can be seen that the tsunami waveform $\eta / \zeta_{0}$ has two large peaks of comparable amplitudes, one in the front of the block due to sliding of the block forward, and the other one behind the block due to spreading of the depletion zone. These results are in good agreement with the aspect of the tsunami waveforms generated by a slowly spreading slump and slide of the ocean bottom presented by Todorovsk et al. [9] and Hayir [28] who considered very simple kinematic source models represented by sliding Heaviside step 

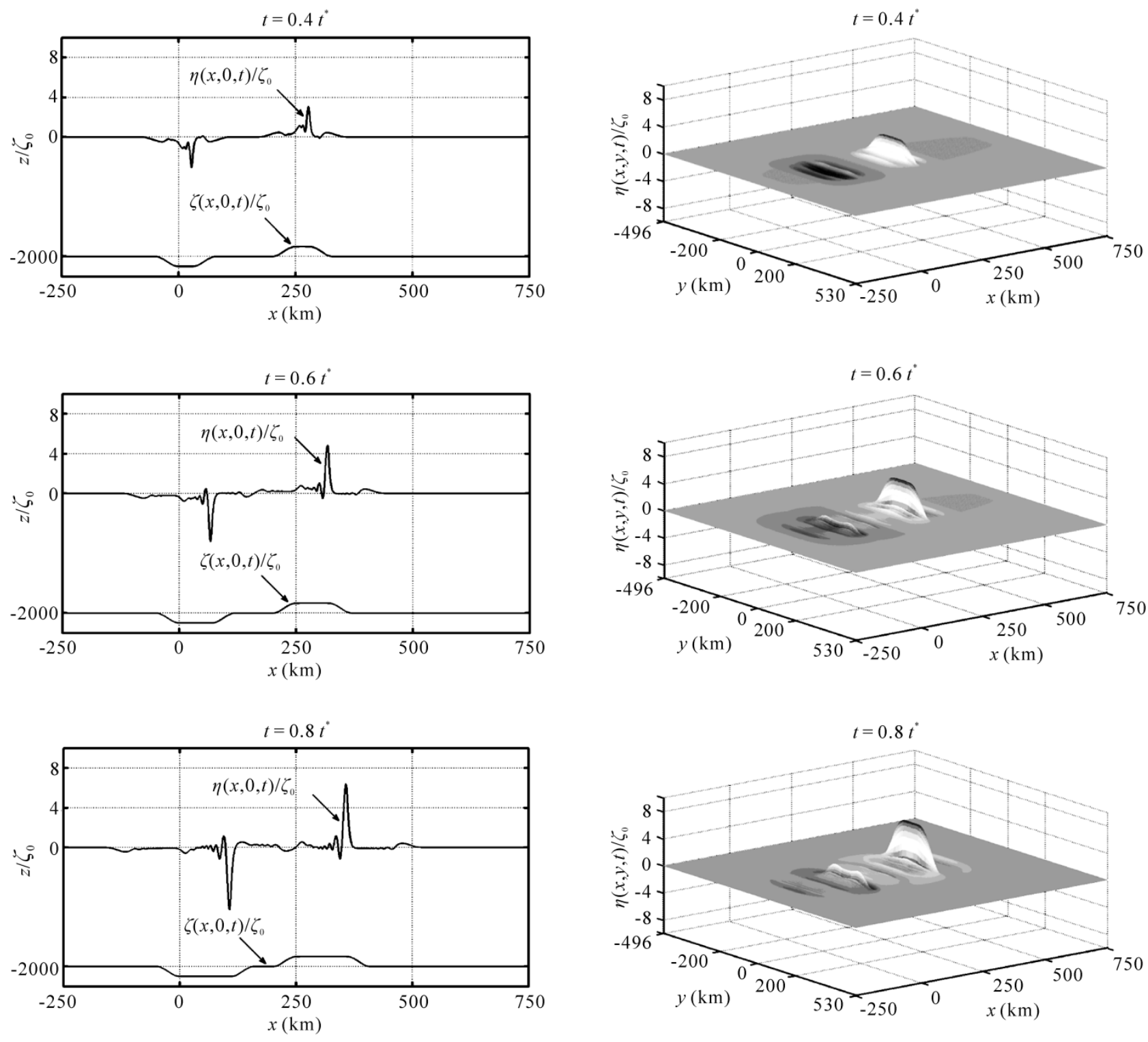

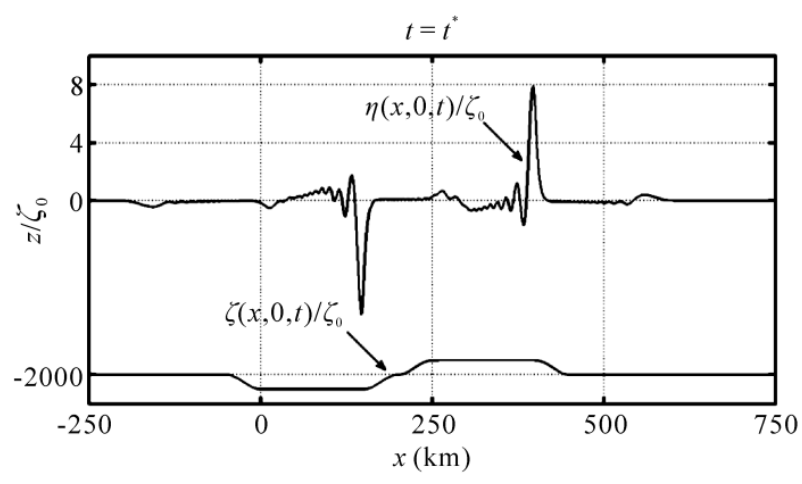

(a)

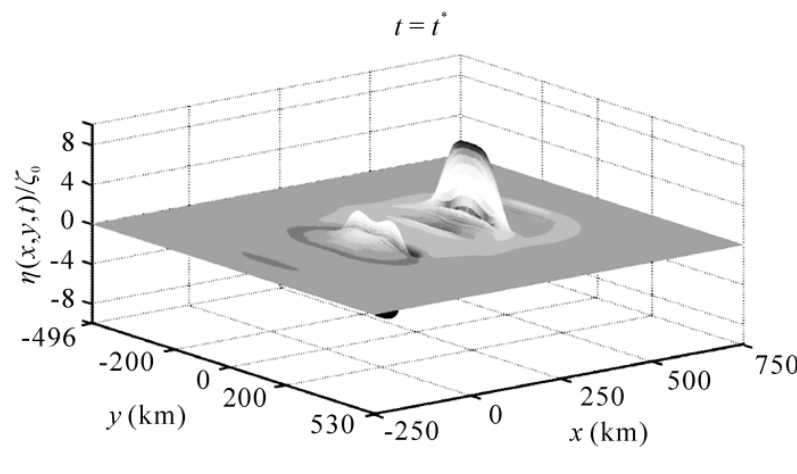

(b)

Figure 6. Dimensionless free-surface elevation caused by the propagation of the slump and slide in the $x$-direction during the second stage with $v=v_{t}$ at $h=2 \mathrm{~km}, L=150 \mathrm{~km}, W=100 \mathrm{~km}$ and $t^{*}=200 / v$ sec; (a) Side view along the axis of symmetry at $y=0$; (b) Three-dimensional view. 
functions. It is difficult to estimate, at present, how often this type of amplification may occur during actual slow submarine process, because of the lack of detailed knowledge about the ground deformations in the source area of past tsunamis. Therefore, we presented here only the basic ideas and illustrated the possible range of amplification factors by means of a realistic curvilinear slip-fault models.

Figures $\mathbf{7}$ and $\mathbf{8}$ illustrate the normalized peak tsunami amplitudes $\eta_{R, \max } / \zeta_{0},\left|\eta_{L, \min }\right| / \zeta_{0}$ respectively in the near-field versus $L / h$ at $t=t^{*}=t_{1}+L / v$, the time when the spreading of the slides stops for $h=0.5,1,1.5$ and $2 \mathrm{~m}$ and for $v=v_{t}$ and $L=150 \mathrm{~km}, W=100 \mathrm{~km}$.

From Figures 7 and 8, the parameter that governs the amplification of the near-field water waves by focusing, is the ratio $L / h$. As the spreading length $L$ in the slip-faults increases, the amplitude of the tsunami wave becomes higher. At $L=0$, no propagation occurs and the waveform takes initially the shape and amplitude of the curvilinear uplift fault (i.e. $\eta_{R, \max } / \zeta_{0}=1, \eta_{L, \min } / \zeta_{0}=1$ ) The negative peak wave amplitudes are approximately equal to the positive peak amplitudes $\left(\eta_{L, \min } / \zeta_{0} \approx\right.$ $\left.\eta_{R, \max } / \zeta_{0}\right)$ when $v=v_{t}$ as seen in Figures 7 and 8 . The peak tsunami amplitude also depends on the water depth in the sense that even a small area source can generate large amplitude if the water is shallow.

Figure 9 shows the effect of the water depth $h$ on the amplification factor $\eta_{R, \max } / \zeta_{0}$ for $v=v_{t}$, with $L=W=$ $10,50,100 \mathrm{~km}$ and $L=150 \mathrm{~km}, W=100 \mathrm{~km}$ at the end of the second stage (i.e. at $t=t^{*}=t_{1}+L / v$ ). Normalized maximum tsunami amplitudes for 19 ocean depths are calculated. As seen from Figure 9, the amplification

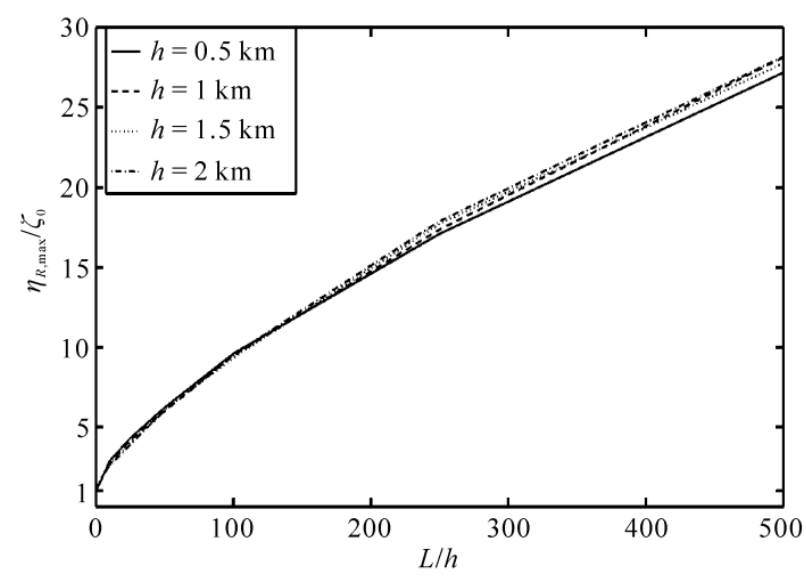

Figure 7. Normalized tsunami peak amplitudes, $\eta_{R, \max } / \zeta_{0}$ at the end of second stage for different water depths $\boldsymbol{h}=\mathbf{0 . 5}$, $1,1.5$ and $2 \mathrm{k}$ at $t^{*}=t_{1}+L / v$ with $v=v_{t}$ and $L=150 \mathrm{~km}$, $W=100 \mathrm{~km}$.

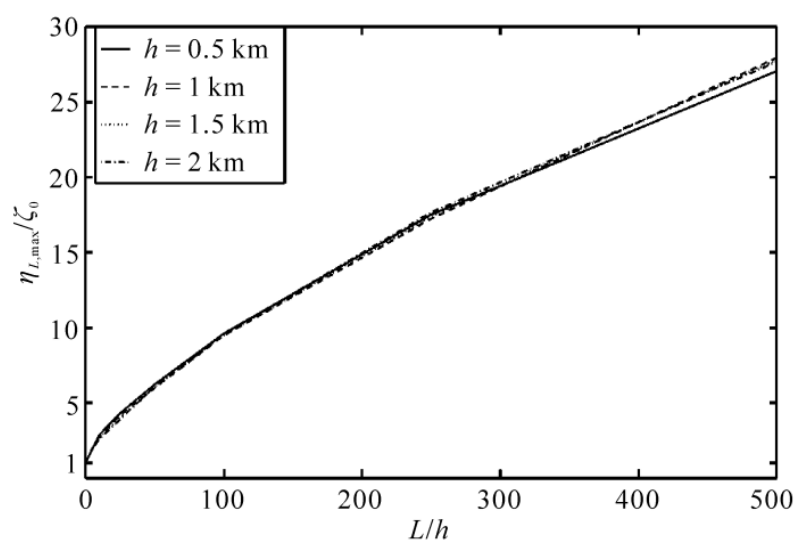

Figure 8. Normalized tsunami peak amplitudes, $\left|\eta_{L, \min }\right| / \zeta_{0}$ at the end of second stage for different water depths $h=0.5$, $1,1.5$ and $2 \mathrm{k}$ at $t^{*}=t_{1}+L / v$ with $v=v_{t}$ and $L=150 \mathrm{~km}$, $W=100 \mathbf{k m}$.

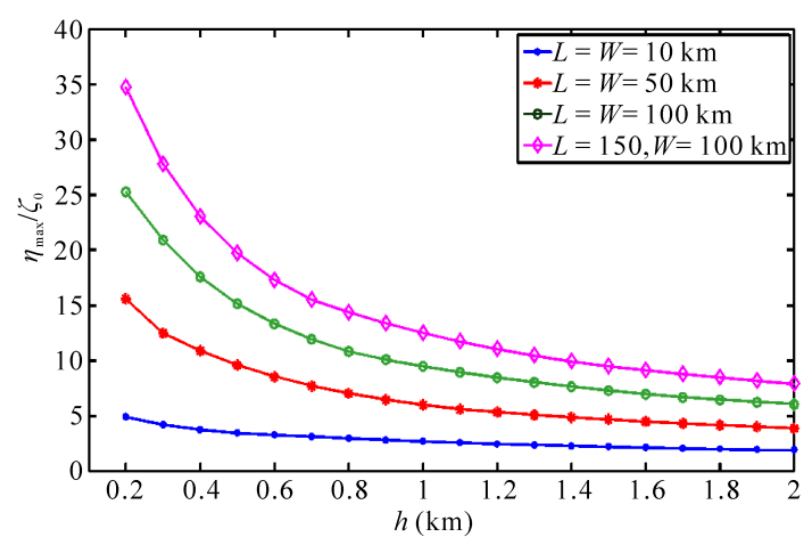

Figure 9. Normalized maximum tsunami amplitudes $\eta_{R, \max } / \zeta_{0}$ for different lengths and widths at $t=t^{*}=$ $t_{1}+L / v$ for $v=v_{t}$.

factor $\eta_{R \text {, max }} / \zeta_{0}$ decreases as the water depth h increases. This happens because the speed of the tsunami is related to the water depth $\left(v=v_{t}=\sqrt{g h}\right)$ which produces small wavelength as the velocity decreases and hence the height of the wave grows as the change of total energy of the tsunami remains constant. Mathematically, wave energy is proportional to both the length of the wave and the height squared.

Therefore, if the energy remains constant and the wavelength decreases, then the height must increase. The results shown in Figures 7-9 agree with the results obtained by Hayir (see Figures 5(b) and 1(b) in [40]) who determined the effects of ocean depth on tsunami amplitudes for very simple kinematic source models represented by a Heaviside step function, since the ratio $L / h$ and the ocean depth have primary effects on normalized peak tsunami amplitudes. 


\subsection{Tsunami Generation and Propagation-Effect of Variable Velocities of Submarine Block Slide}

In this section, we investigated the motion of a submarine block slide, with variable velocities, and its effect on the near-field tsunami amplitudes. We considered the limiting case, in which the slide moves with constant velocity and stops suddenly (infinite deceleration) and the case in which the slide stops softly with constant deceleration for $L=150 \mathrm{~km}, W=100 \mathrm{~km}$ and $v=v_{t}$.

\subsubsection{Displaced Block Sliding with Constant Velocity $v$}

Constant velocity implies that the slide starts and stops impulsively, i.e. the acceleration and deceleration are infinite both initially and finally. This means that the slide takes minimum time to reach the characteristic length $L^{\prime}=150 \mathrm{~km}$ given by $t_{\text {min }}=t^{*}+\left(L^{\prime} / v\right)=41.65$ min. We illustrated the impulsive tsunami waves caused by sudden stop of the slide at distance $L^{\prime}$ in Figure 10.

Figure 10 shows the leading tsunami wave propagating in the positive $\mathrm{x}$-direction during time evolution $t=t^{*}, t^{*}+0.2\left(L^{\prime} / v\right), t^{*}+0.4\left(L^{\prime} / v\right), t^{*}+0.6\left(L^{\prime} / v\right)$, $t^{*}+0.8\left(L^{\prime} / v\right), t^{*}+\left(L^{\prime} / v\right)$ ec at. $L_{E}=0,30,60,90$, $120,150 \mathrm{~km}$ respectively, where $L_{E}$ represents that part of $L$ (see Figure 4(c)) for $v=v_{t}=0.14 \mathrm{~km} / \mathrm{sec}$. It is seen in Figure $\mathbf{1 0}$ that the maximum leading wave amplitude decreases with time, due to the geometric spreading and also due to the dispersion. At $t=t_{\min }=$ $t^{*}+\left(L^{\prime} / v\right)=41.65$, the wave front is at $x=693 \mathrm{~km}$ and $\eta_{R, \max } / \zeta_{0}$ decreases from 7.906 at $t=t^{*}$ to 5.261 at time $t_{\min }$. This happens because the amplification of the waveforms depends only on the volume of the displaced water by the moving source which becomes an important factor in the modeling of the tsunami generation. This was clear from the singular points removed from the block slide model, where the finite limit of the free surface depends on the characteristic volume of the source model. This result agrees with the results obtained by Guard et al. [41] who studied the tsunami wave generation caused by a simple seabed deformation represented by a translating hump that moves with constant velocity. It has seen in their results that the tsunami amplitudes are reduced with time when the hump moves with constant velocity and that the characteristic wavelength was increased with the increase in the water depth.

\subsubsection{Displaced Block Slide Moving with Linear Decreasing Velocity with Time $T$}

The velocity of the movable slide is uniform and equal $v(t)$ up to time $t_{\max }$ as shown in Figure 4(a), followed by a decelerating phase in which the velocity is given by $v(t)=v-\left(t-t^{*}\right) \alpha$, for $t^{*} \leq t \leq t_{\max }$. where $v=v_{t}=$ $0.14 \mathrm{~km} / \mathrm{sec}$ and $\alpha$ is the deceleration of the moving block slide. The block slide moves in the positive x-direction with time $t^{*} \leq t \leq t_{\max }$ where $t_{\max }=t^{*}+$ $2 L^{\prime} / v$ is the maximum time that the slide takes to stop after reaching the characteristic length $L^{\prime}=150 \mathrm{~km}$ with minimum deceleration $\alpha_{\min }$. Figure 11 shows the leading tsunami wave propagating in the positive $\mathrm{x}$-direction during time evolution $t=t^{*}, t^{*}+0.2\left(L^{\prime} / v\right)$, $t^{*}+0.4\left(L^{\prime} / v\right), \quad t^{*}+0.6\left(L^{\prime} / v\right), \quad t^{*}+0.8\left(L^{\prime} / v\right), \quad t^{*}+$ $\left(L^{\prime} / v\right) \min$ in case $t_{2}=t^{*}$ (i.e. minimum magnitude of $\alpha)$.

It is clear from Figures $\mathbf{1 0}$ and $\mathbf{1 1}$ that at the instant the slide stops, the peak amplitude in case of sudden stop is higher than that of soft stop.

Figures 12 and $\mathbf{1 3}$ show the effect of the water depth at $L^{\prime}=W 10,50,100 \mathrm{~km}$ and $L^{\prime}=150, W=100 \mathrm{~km}$ on the normalized peak tsunami amplitude $\eta_{R, \max } / \zeta_{0}$ when the slide stops moving at length $L^{\prime}$ instantaneously at $t_{\min }=t^{*}+\left(L^{\prime} / v\right)$ with infinite deceleration and stops moving softly at $L^{\prime}$ at the time $t_{\max }=t^{*}+$ $\left(2 L^{\prime} / v\right)$ with minimum deceleration for $v=v_{t}$.

It is clear from Figures $\mathbf{1 2}$ and $\mathbf{1 3}$ that the waveforms which are caused by sudden stop of the slide motion after

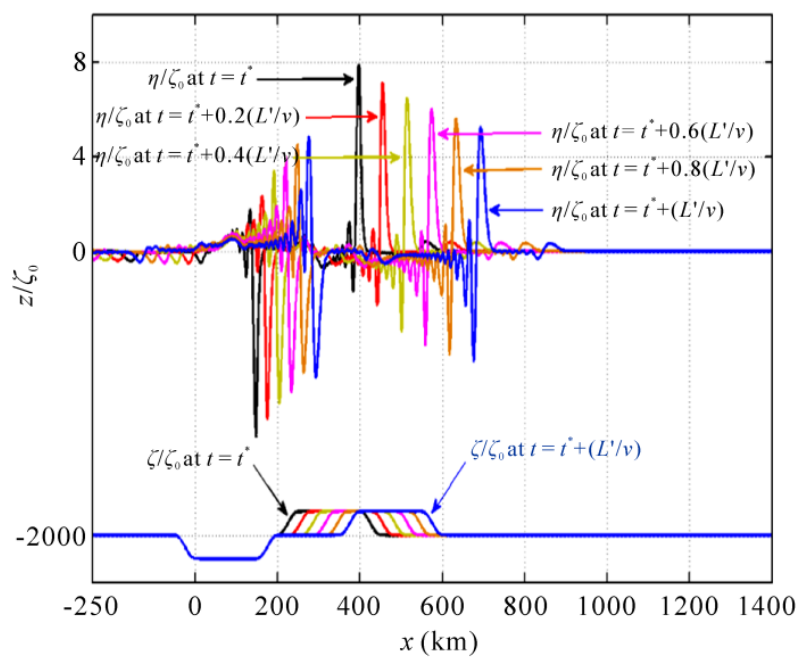

Figure 10. Normalized tsunami waveforms $\eta / \zeta_{0}$ along the axis of symmetry at $\boldsymbol{y}=\mathbf{0}$ and their corresponding moving slide $\zeta / \zeta_{0}$ with constant velocity $v$ along $y=0$, at time $t^{*} \leq t \leq t^{*}+\left(L^{\prime} / v\right)$ for $h=2 \mathrm{~km}, L^{\prime}=150 \mathrm{~km}$ and $W=$ $100 \mathrm{~km}$. 


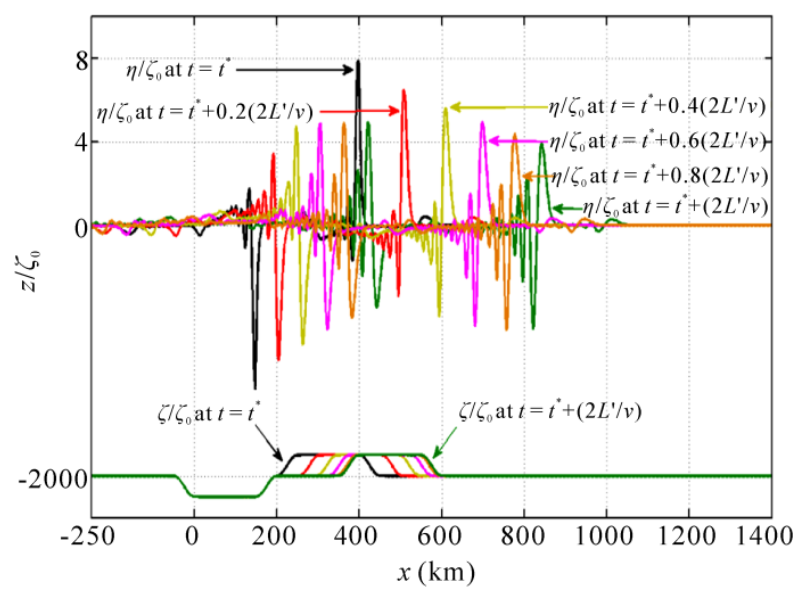

Figure 11. Normalized tsunami waveforms $\eta / \zeta_{0}$ and their corresponding moving slide $\zeta / \zeta_{0}$ with variable velocity $v(t)$ along $y=0$, at time $t^{*} \leq t \leq t^{*}+\left(2 L^{\prime} / v\right)$ at $h=2 \mathrm{~km}$, $L^{\prime}=150 \mathrm{~km}$ and $W=100 \mathrm{~km}$.

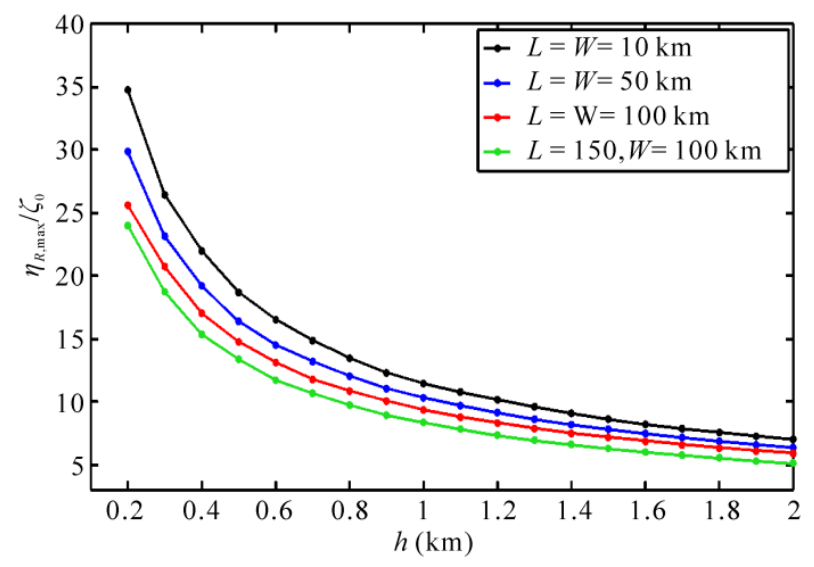

Figure 12. Normalized maximum tsunami amplitudes $\eta_{R, \max } / \zeta_{0}$ when the slide stops suddenly at time $t_{\min }=$ $t^{*}+\left(L^{\prime} / v\right)$ with different slide lengths $L$ and widths $W$ and for $v=v_{t}$.

they reach the characteristic length $L^{\prime}$ at time $t_{\min }=$ $t^{*}+\left(L^{\prime} / v\right)$ have higher amplitude than stopping of the slide with slow motion at time $t_{\max }=t^{*}+\left(2 L^{\prime} / v\right)$. This agrees with the mathematical relation between the wavelength and the wave height where the wave energy is proportional to both the length of the wave and the height squared.

\subsubsection{Displaced Block Slide Moving with Constant Velocity $v$ Followed by Variable Velocity $v(t)$}

In this section, we studied the generation of the tsunami waveforms when the block slide moves a significant distance with constant velocity $v=v_{t}$ then continues moving

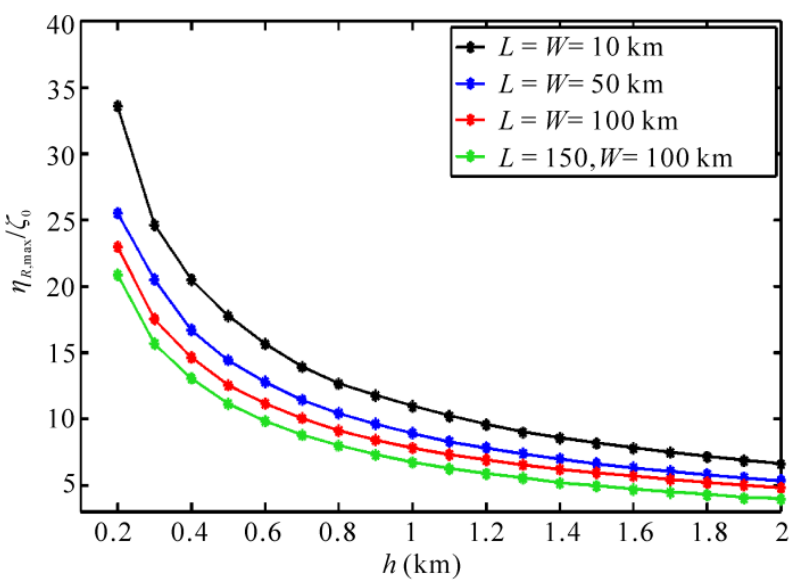

Figure 13. Normalized maximum tsunami amplitudes $\eta_{R, \max } / \zeta_{0}$ when the slide stops softly at time $t_{\max }=$ $t^{*}+\left(2 L^{\prime} / v\right)$ with different slide lengths $L$ and widths $W$ and for $v=v_{t}$.

with variable velocity $v(t)$ with constant deceleration until it stops at the characteristic length $L^{\prime}=150 \mathrm{~km}$. Figure 14 shows the tsunami waveforms at the times calculated in Table 2 when the slide reaches the characteristic length $L^{\prime}=150 \mathrm{~km}$.

In Figure 14, the blue waveform indicates the shape of the wave at the time $t_{3}=t_{\min }=t^{*}+\left(L^{\prime} / v\right)=41.65$ min in the limiting case when the slide stops moving suddenly. The green waveform indicates the wave in the other limiting case at the time $t_{3}=t_{\max }=t^{*}+\left(2 L^{\prime} / v\right)$ when the slide stops moving with minimum deceleration at the distance $L^{\prime}$. In between the two limiting cases, the slide begins moving with constant velocity a significant distance followed by decelerating movement until it stops at the characteristic length $L^{\prime}=150 \mathrm{~km}$ at the time $t_{\min } \leq t_{3} \leq t_{\max }$, see Table 2. It is seen how the peak amplitudes of the leading waves decreases gradually from 5.261 to 3.894 .

In order to compare the shape and maximum height of tsunami wave at certain time for different deceleration $\alpha$, we choose the time $t=t_{\max }$. For the limiting case $\alpha_{\text {min }}$, there is no free propagation, while for the other limiting case "the sudden stop", there is maximum free propagation between time $t_{\min }$ and $t_{\max }$. For the cases between the two limiting cases, the propagation time is $t_{\text {prop }}=t_{\max }-t_{3}$.

Figure 15 shows the shape of the tsunami propagation waveform at $t_{\max }=t^{*}+\left(2 L^{\prime} / v\right)=59.51 \mathrm{~min}$ (curves in black) for different deceleration $\alpha$ and time $t_{3}$ (time at which the slide stops). As the wave propagates, the wave height decreases and the slope of the wave front 


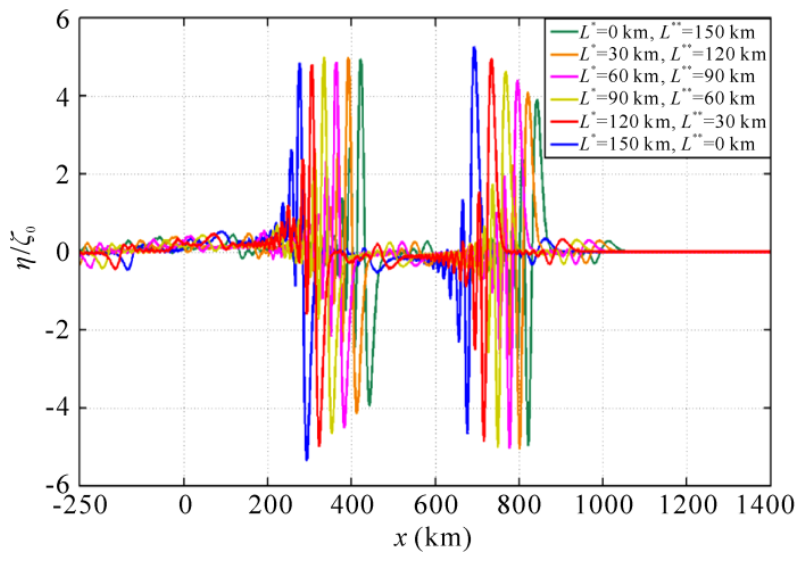

Figure 14. Normalized tsunami waveforms $\eta / \zeta_{0}$ along $y=$ 0 at $t=t_{3}$ calculated in Table 2 with $L^{\prime}=150 \mathrm{~km}$.

becomes smaller, causing a train of small wave forms behind the main wave. The maximum wave amplitude decreases with time, due to the geometric spreading and also due to the dispersion.

Figure 16 represents the normalized peak tsunami amplitudes $|\eta|_{\min } / \zeta_{0}$ and $\eta_{\max } / \zeta_{0}$ of the leading propagating wave in the far-field at the time $t=t_{\max }$ for the different deceleration $\alpha$ and time $t_{3}$ (time at which the slide stops) chosen in Figure 15.
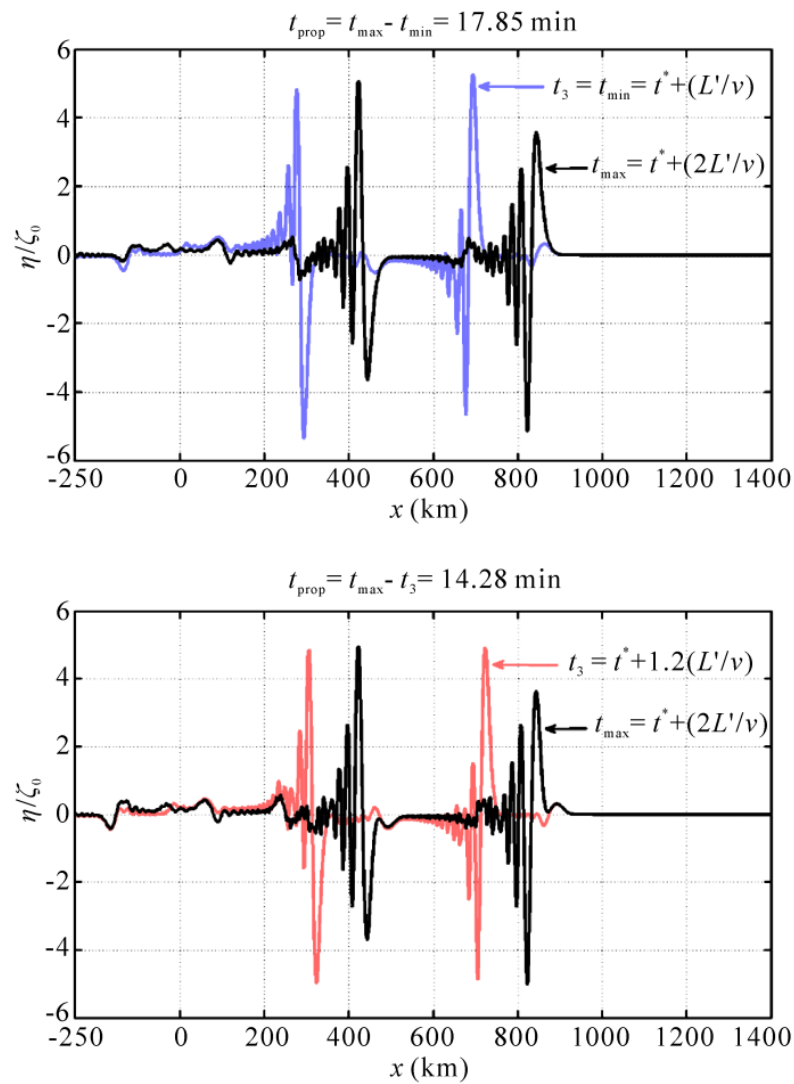
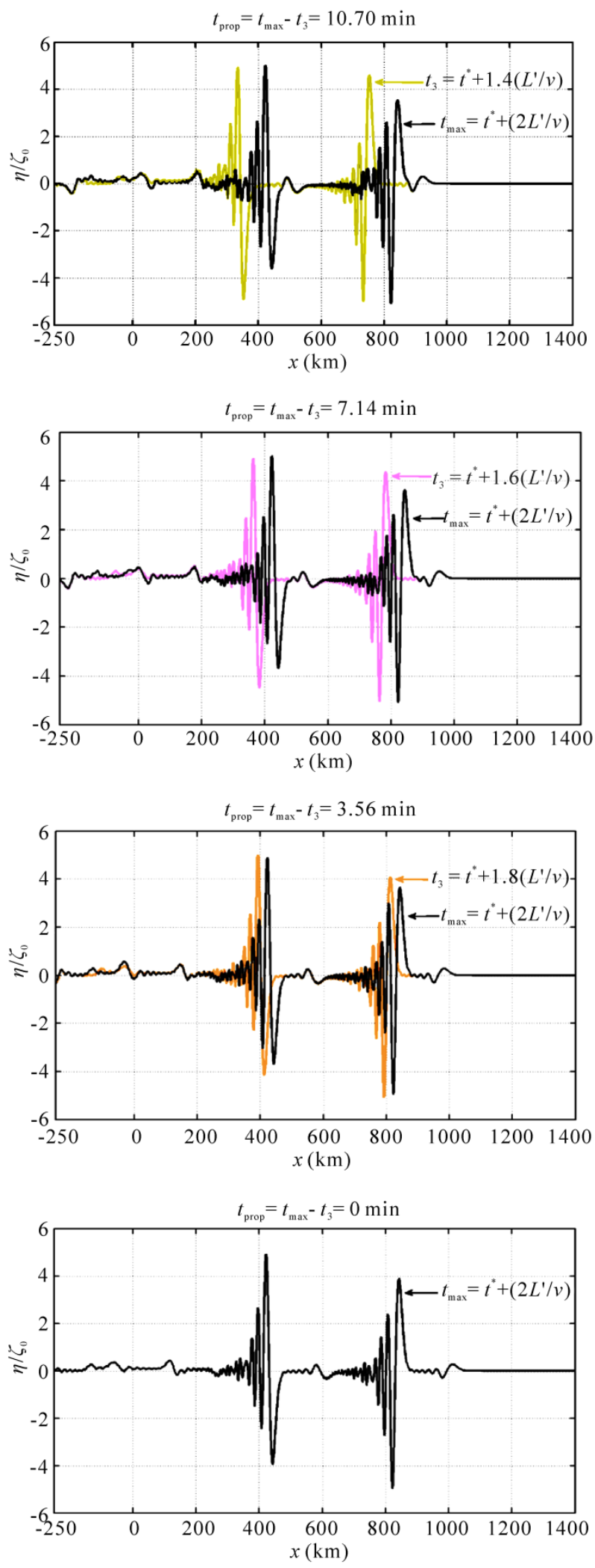

Figure 15. Normalized tsunami propagation waveforms $\eta / \zeta_{0}$ along the axis of symmetry at $y=0$ at time $t_{\max }=$ $59.51 \mathrm{~min}$ and at time $t_{3}$ for different deceleration $\alpha$. 


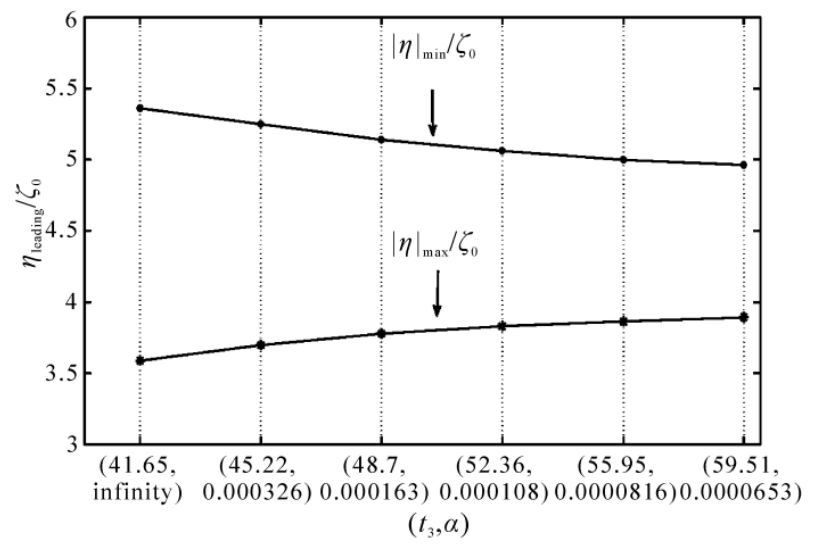

Figure 16. The normalized peak tsunami amplitudes $|\eta|_{\min } / \zeta_{0}$ and $\eta_{\max } / \zeta_{0}$ at time $t=t_{\max }$ for the different deceleration $\alpha$ and time $t_{3}$.

It can be seen in Figure 16 that the absolute minimum peak amplitudes of the leading propagated waves at time $t_{\max }=t^{*}+\left(2 L^{\prime} / v\right)$, after the block slide stops moving with different deceleration $\alpha$ and time $t_{3}$, decrease gradually, while the maximum peak amplitudes increase progressively.

\section{Conclusions}

In this paper, we presented a review of the main physical characteristics of the tsunami generation caused by realistic curvilinear submarine slumps and slides in the near-field. It is seen that the tsunami waveform has two large peaks of comparable amplitudes, one in the front of the block due to forward sliding of the block, and the other one behind the block due to spreading of the depletion zone. The negative peak wave amplitudes are approximately equal to the positive peak amplitudes. These results agree with the qualitative behaviour of the tsunami waveforms generated by a slowly spreading slump and slide of the ocean bottom presented by Todorovsk et al. [9] and Hayir [28] who considered very simple kinematic source models represented by sliding Heaviside step functions. We studied the effect of variable velocities of submarine block slide on the tsunami generation in the limiting cases, in which the slide moves with constant velocity and stops suddenly (with infinite deceleration) and the case in which the slide stops softly at the same place with minimum deceleration. It is seen that the leading tsunami amplitudes are reduced in both cases due to the geometric spreading and also due to the dispersion. We observed that the peak tsunami amplitudes increase with the decrease in the sliding source area and the water depth. We also investigated the more realistic case in which the block slide moves a significant distance with constant velocity $v$ then continue moving with time de- pendence velocity $v(t)$ and different constant deceleration until it stops at the characteristic length. It is seen how the peak amplitudes of the leading waves decrease gradually with time between the two limiting cases. In this case we demonstrated also the shape of tsunami propagated wave at certain time $t_{\max }$ (time at which the slide stops with minimum deceleration). The results show that the wave height decreases due to dispersion and the slope of the front of the wave becomes smaller, causing a train of small wave forms behind the main wave. It can be observed that just a slight variation in the maximum and the minimum tsunami propagated amplitudes after the block slide stops moving with different deceleration $\alpha$ and time $t_{3}$, see Figure 16. The presented analysis suggests that some abnormally large tsunamis could be explained in part by variable speeds of submarine landslides. Our results should help to enable quantitative tsunami forecasts and warnings based on recoverable seismic data and to increase the possibilities for the use of tsunami data to study earthquakes, particularly historical events for which adequate seismic data do not exist.

\section{Acknowledgments}

The authors would like to express their gratitude to Professor Mina B. Abd-el-Malek, head of the Department of Engineering Mathematics and Physics, Faculty of Engineering, Alexandria University, Egypt for his valuable suggestions and discussions which led to many improvements of the manuscript. The authors would like to thank the reviewer for his/her valuable comments, which improved the paper.

\section{References}

[1] M. S. Abou-Dina and F. M. Hassan, "Generation and Propagation of Nonlinear Tsunamis in Shallow Water by a Moving Topography," Applied Mathematics and Computation, Vol. 177, No. 2, 2006, pp. 785-806.

[2] F. M. Hassan, "Boundary Integral Method Applied to the Propagation of Non-linear Gravity Waves Generated by a Moving Bottom," Applied MathematicalModelling, Vol. 33, No. 1, 2009, pp. 451-466.

[3] N. Zahibo, E. Pelinovsky, T. Talipova , A. Kozelkov and A. Kurkin, "Analytical and Numerical Study of Nonlinear Effects at Tsunami Modeling," Applied Mathematics and Computation, Vol. 174, No. 2, 2006, pp. 795-809.

[4] K. Das, S. Green, D. Basu, R. Janetzke and J. Stamatakos, "Effect of Slide Deformation and Geometry on Waves Generated by Submarine Landslides: A Numerical Investigation," Offshore Technology Conference, Texas, USA, May 2009, pp. 1-12.

[5] Ben-Menahem and M. Rosenman, “Amplitude Patterns 
of Tsunami Waves from Submarine Earthquakes," Journal of Geophysical Research, Vol. 77, No. 17, 1972, pp. 3097-3128.

[6] M. I. Todorovska and M. D. Trifunac, "Generation of Tsunamis by a Slowly Spreading Uplift of the Sea Floor," Soil Dynamics and Earthquake Engineering, Vol. 21, No. 2, 2001, pp. 151-167.

[7] M. D. Trifunac and M. I. Todorovska, "A Note on Differences in Tsunami Source Parameters for Submarine Slides and Earthquakes," Soil Dynamics and Earthquake Engineering, Vol. 22, No. 2, 2002, pp.143-155.

[8] M. D. Trifunac, A. Hayir and M. I. Todorovska, “A Note on Tsunami Caused by Submarine Slides and Slumps Spreading in One Dimension with Nonuniform Displacement Amplitudes," Soil Dynamics and Earthquake Engineering, Vol. 23, No. 3, 2003, pp. 223-234.

[9] M. I. Todorovsk, M. D. Trifunac and A. Hayir, "A Note on Tsunami Amplitudes above Submarine Slides and Slumps," Soil Dynamics and Earthquake Engineering, Vol. 22, No. 2, 2002, pp. 129-141.

[10] M. D. Trifunaca, A. Hayira and M. I. Todorovska, "Was Grand Banks Event of 1929 a Slump Spreading in Two Directions," Soil Dynamics and Earthquake Engineering, Vol. 22, No. 5, 2002, pp. 349-360.

[11] Hayir, "The Near-Field Tsunami Amplitudes Caused by Submarine Landslides and Slumps Spreading in Two Prthogonal Directions," Ocean Engineering, Vol. 33, No. 5-6, 2006, pp. 654-664.

[12] S. A. Beisel, L. B. Chubarov, Z. I. Fedotova and G. S. Khakimzyanov, "On the Approaches to a Numerical Modeling of Landslide Mechanism of Tsunami Wave Generation," Communications in Applied Analysis, Vol. 11, No. 1, 2007, pp. 121-135.

[13] P. Lynett and L.-F. Liu Philip, "A Numerical Study of Submarine-Landslide-Generated Waves and Run-Up," Proceeding of the Royal Society A, Vol. 458, No. 2028, 2002, pp. 2885-2910.

[14] L. Jiang and P. H. Le Blond, "The Coupling of a Submarine Slide and the Surface Waves Which it Generates," Journal of Geophysical Research, Vol. 97, No. C8, 1992, pp. 12731-12744.

[15] S. Rzadkiewicz, C. Mariotti and P. Heinrich, "Numerical Simulation of Submarine Landslides and Their Hydraulic Effects," Journal of Waterway, Port, Coastal, and Ocean Engineering, Vol. 123, No. 4, 1997, pp. 149-157.

[16] S. T. Grilli and P. Watts, "Modeling of Waves Generated by a Moving Dubmerged Body, Applications to Underwater Landslides," Journal of Engineering Analysis with Boundary Elements, Vol. 23, No. 8, pp. 1999, 645-656.

[17] P. Watts, S. T. Grilli, D. R. Tappin and G. J. Fryer, "Tsunami Generation by Dubmarine Mass Failure. II: Predictive Equations and Case Studies," Journal of Waterway, Port, Coastal, and Ocean Engineering, Vol. 131, No. 6, 2005, pp. 298-310.

[18] B. Ataie-Ashtiani and G. Shobeyri, "Numerical simulation of landslide impulsive waves by incompressible smoothed particle hydrodynamics," International Journal for Numerical Methods in Fluids, Vol. 56, No. 2, 2008, pp. 209-232.

[19] R. Agustinus, "Numerical Study of Tsunami Waves with Sloping Bottom and Nonlinear Friction," Journal of Mathematics and Statistics, Vol. 4, No. 1, 2008, pp. 41-45.

[20] Najafi-Jilani and B. Ataie-Ashtiani, "Estimation of Near-Field Characteristics of Tsunami Generation by Submarine Landslide," Ocean Engineering, Vol. 35, No. 5-6, 2008, pp. 545- 557.

[21] P. Watts, "Wave Maker Curves for Tsunamis Generated by Underwater Landslides," Journal of Waterway, Port, Coastal, and Ocean Engineering, Vol. 124, No. 3, 1998 , pp. 127-137.

[22] P. Watts and S. T. Grilli, "Underwater Landslide Shape, Motion, Deformation, and Tsunami Generation," Proceedings of The Thirteenth Internation Offshore and Polar Engineering Conference, Honolulu, Hawaii, USA, May 25-30, 2003, pp. 364-371.

[23] H. M. Fritz, W. H. Hager and H. E. Minor, "Near Field Characteristics of Landslide Generated Impulse Waves," Journal of Waterway, Port, Coastal, and Ocean Engineering, Vol. 130, No. 6, 2004, pp. 287-302.

[24] B. Ataie-Ashtiani and A. Najafi-Jilani, "Higher-order Boussinesq-Type Model with Moving Bottom Boundary: Applications to Submarine Landslide Tsunami Waves," International Journal for Numerical Methods in Fluids, Vol. 53, No. 6 2007, pp. 1019-1048.

[25] E. François and T. G. Stephan, "Experimental Study of Tsunami Generation by Three-Dimensional Rigid Underwater Landslides," Journal of Waterway, Port, Coastal, and Ocean Engineering, Vol. 133, No. 6, 2007, pp. $442-454$

[26] S. N. Ward, "Landslide Tsunami," Journal of Geophysical Research, Vol. 106, No. B6, 2001, pp. 201-215.

[27] M. D. Trifunac, A. Hayir and M. I. Todorovska, "A Note on the Effects of Nonuniform Spreading Velocity of Submarine Slumps and Slides on the Near-Field Tsunami Amplitudes," Soil Dynamics and Earthquake Engineering, Vol. 22, No. 3, 2002, pp. 167-180.

[28] Hayir, "The Effects of Variable Speeds of a Submarine Block Slide on Near-field Tsunami Amplitudes," Ocean Engineering, Vol. 30, No. 18, 2003, pp. 2329-2342.

[29] V. V. Titov and F. I. Gonzalez, "Implementation and Testing of the Method of Splitting Tsunami (MOST) Model," NOAA/Pacific Marine Environmental Laboratory, U.S., 1997.

[30] A.Yu. Bezhaev, M.M. Lavrentiev and An.G. Marchuk, V. V. Titov, "Determination of Tsunami Sources Using Deep Ocean Wave Records," Bull. Nov. Comp. Center Math. Model in Geoph., Vol. 11, No. 2006, pp. 53-63.

[31] L. Jordanovski and M. I. Todorovska, "Inverse Studies of the Earthquake Source Mechanism from Near-Field Strong Motion Records," Journal of Indian Society of Earthquake Technology, Vol. 39, No. 1-2, 2002, pp. 73-91.

[32] D. R. Fuhrman and Per. A. Madsen, "Tsunami Generation, Propagation, and Run-Up with a High-Order Bous- 
sinesq Model," Coastal Engineering, Vol. 56, No. 7, pp. 2009, 747-758.

[33] Xi Zhao, B. Wang and H. Liu, "Modeling the Submarine Mass Failure Induced Tsunamis by Boussinesq Equations," Journal of Asian Earth Sciences, Vol. 36, No. 1, 2009, pp. 47-55.

[34] L. Hammack, "A Note on Tsunamis: their Generation and Propagation in an Ocean of Uniform Depth," Journal of Fluid Mechanics, Vol. 60, No. 4, 1973, pp. 769-799.

[35] D. Dutykh, F. Dias, "Water Waves Generated by a Moving Bottom," In: A. Kundu Ed., Tsunami and Nonlinear Waves, Geosciences, Springer Verlag, 2007, pp. 65-95.

[36] H. S. Hassan, K. T. Ramadan and S. N. Hanna, "Modeling of Tsunami Generation and Propagation by a Spreading Curvilinear Seismic Faulting in Linearized Shallow-Water Wave Theory," accepted for publication.

[37] W. P. Dillon, J. S. Risch, K.M. Scanlon, P. C. Valentine and T. Higgett, "Ancient Crustal Fractures Control the Location and Size of Collapsed Blocks at the Blake Rscarpment," east of Florida. Submarine Landslides: Selected Studies in the US Exclusive Economic Zone. US
Geological Survey Bulletin 2002, US Department of the interior, Denver, 1993, pp. 54-68.

[38] P. R. Carlson, H. A. Karl, B. D. Edwards, J. V. Gardner and K. Hall, "Mass Movement Related to Large Submarine Canyons along the Beringian Margin, Alaska. Submarine Landslide," Selected Studies in the US Exclusive Economic Zone, US Geological Survey Bullettin 2002, US Department of Interior, Denver, 1993, pp. 104-116.

[39] C. E. Gutmacher and W. RNormark, "Sur Submarine Landslide, a Deep-Water Sediment Slope Failure. Submarine Landslides," Selected Studies in the US Exclusive Economic Zone. US Geological Survey Bulletin 2002, US Depth of the interior, Denver, 1993, pp. 158- 166.

[40] Hayir, "Ocean Depth Effects on Tsunami Amplitudes Used in Source Models in Linearized Shallow-Water Wave Theory," Ocean Engineering, Vol. 31, No. 3-4, 2004, pp. 353-361.

[41] P. A. Guard and P. Nielsen, "A Simple Model for Tsunami Wave Due to Submarine Landslides," International Coastal Symposium, Session, 5, Republic of Iceland, 2005. 